\title{
On Weddle Surfaces And Their Moduli
}

\author{
Michele Bolognesi
}

\begin{abstract}
The Weddle surface is classically known to be a birational (partially desingularized) model of the Kummer surface. In this note we go through its relations with moduli spaces of abelian varieties and of rank two vector bundles on a genus 2 curve. First we construct a moduli space $\mathcal{A}_{2}(3)^{-}$parametrizing abelian surfaces with a symmetric theta structure and an odd theta characteristic. Such objects can in fact be seen as Weddle surfaces. We prove that $\mathcal{A}_{2}(3)^{-}$is rational. Then, given a genus 2 curve $C$, we give an interpretation of the Weddle surface as a moduli space of extensions classes (invariant with respect to the hyperelliptic involution) of the canonical sheaf $\omega$ of $C$ with $\omega^{-1}$. This in turn allows to see the Weddle surface as a hyperplane section of the secant variety $\operatorname{Sec}(C)$ of the curve $C$ tricanonically embedded in $\mathbb{P}^{4}$.
\end{abstract}

\section{Introduction}

The Burkhardt quartic hypersurface $\mathcal{B} \subset \mathbb{P}^{4}$ is a hypersurface defined by the vanishing of the unique $S p(4, \mathbb{Z} / 3 \mathbb{Z}) / \pm I d$ invariant quartic polynomial. Its explicit equation was written down for the first time by $\mathrm{H}$. Burkhardt in 1892 [Bur92]. It was probably known to Coble (or at least one can infer that from his results) that a generic point of $\mathcal{B}$ represents a principally polarized abelian surface (ppas for short) with a level 3 structure but it was only recently that G. Van der Geer [vdG87] made this statement clearer. In particular Van der Geer ( [vdG87], Remark 1) pointed out the fact that the Hessian variety Hess $(\mathcal{B})$ of the Burkhardt quartic is birational to the moduli space parametrizing ppas with a symmetric theta structure and an even theta characteristic, which we will denote by $\mathcal{A}_{2}(3)^{+}$. The moduli space $\mathcal{A}_{2}(3)^{+}$is constructed as a quotient of the Siegel upper half space $\mathbb{H}_{2}$ by the arithmetic group $\Gamma_{2}(3,6)$. Moreover, since $\mathcal{B}$ is self-Steinerian ([Hun96], Chapter 5), one can view the 10:1 Steinerian map

$$
S t_{+}: \operatorname{Hess}(\mathcal{B}) \longrightarrow \mathcal{B}
$$

as the forgetful morphism $f: \mathcal{A}_{2}(3)^{+} \rightarrow \mathcal{A}_{2}(3)$ which forgets the symmetric line bundle representing the polarization. This means that the following diagram, where the horizontal arrows $T h^{+}$and $Q$ are birational isomorphisms, commutes.

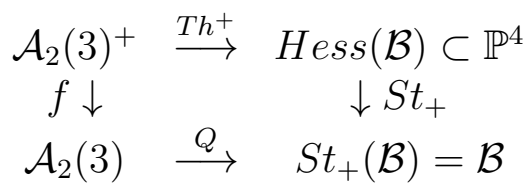

Coble also computed in detail a unirationalization

$$
\pi: \mathbb{P}^{3} \longrightarrow \mathcal{B},
$$


given by a system of quartic polynomials that gives rise to a map of degree 6. By analogy with the Steinerian map (1), the degree of this map has lead us to suspect that $\mathbb{P}^{3}$ could be birational to another moduli space, which we denote by $\mathcal{A}_{2}(3)^{-}$, that should parametrize ppas with a symmetric theta structure and an odd theta characteristic. In this paper we describe the arithmetic group $\Gamma_{2}(3)^{-}$which realizes $\mathcal{A}_{2}(3)^{-}$as a quotient

$$
\mathcal{A}_{2}(3)^{-}=\mathbb{H}_{2} / \Gamma_{2}(3)^{-} \text {. }
$$

Moreover we prove the following theorem.

Theorem 0.0.1 Let $\mathcal{A}_{2}(3)^{-}$be the moduli space of ppas with a symmetric level 3 structure and an odd theta characteristic. The theta-null map $\mathrm{Th}^{-}$given by even theta functions induces a birational isomorphism

$$
T h^{-}: \mathcal{A}_{2}(3)^{-} \longrightarrow \mathbb{P}^{3} \text {. }
$$

Furthermore, the pullback by $\pi$ of tangent hyperplane sections of $\mathcal{B}$ are Weddle quartic surfaces. Let $C$ be a genus 2 curve and $\tau: \xi \mapsto \xi^{-1} \otimes \omega$ the Serre involution on the Picard variety $\operatorname{Pic}^{1}(C)$. Chosen an appropriate linearization for the action of $\tau$ on $\mathcal{O}_{P i c^{1}(C)}(\Theta)$, the Weddle surface $W$ is the image of $\operatorname{Pic}^{1}(C)$ in $\mathbb{P}^{3}=\mathbb{P} H^{0}\left(\operatorname{Pic}^{1}(C), 3 \Theta\right)_{+}^{*}$ (where the plus indicates that we are considering invariant sections). Moreover the surface $W$ is a birational model of the Kummer surface $K^{1}=P i c^{1}(C) / \tau \subset \mathbb{P} H^{0}\left(P i c^{1}, 2 \Theta\right)^{*}$. Given a ppas $A$ with an odd line bundle $L$ representing the polarization (resp. an even line bundle) one can as well obtain a Weddle surface by sending $A$ in the $\mathbb{P}^{3}$ obtained from the eigenspace $H^{0}\left(A, L^{3}\right)_{+}$ (resp. $\left.H^{0}\left(A, L^{3}\right)_{-}\right)$w.r.t. the standard involution $\pm I d$. Since also this Weddle surface is a birational model of the Kummer surface $K:=A / \pm I d \subset \mathbb{P} H^{0}\left(A, L^{2}\right)^{*}$, we go through the construction of the birational map between the two surfaces, proving that it comes (in the odd line bundle case) from a canonical embedding

$$
Q: H^{0}\left(A, L^{2}\right)^{*} \hookrightarrow \operatorname{Sym}^{2} H^{0}\left(A, L^{3}\right)_{+} .
$$

Furthermore, a point of $\mathcal{A}_{2}(6)$ can be associated to such a configuration of surfaces.

In the second (independent) part of the paper we change our point of view: we fix a smooth genus 2 curve $C$ and consider the moduli space $\mathcal{M}_{C}$ of rank two vector bundles on $C$ with trivial determinant. It is well known [NR69] that $\mathcal{M}_{C}$ is isomorphic to $\mathbb{P}^{3}$, seen as the $2 \Theta$ linear series on the Jacobian of $C$ and that the semistable boundary is the Kummer surface $K^{0}=\operatorname{Jac}(C) / \pm I d \subset|2 \Theta|$. The space $\mathbb{P} \operatorname{Ext}^{1}\left(\omega, \omega^{-1}\right) \cong \mathbb{P}^{4}=\left|\omega^{3}\right|^{*}$ parametrizes extensions classes $(e)$ of $\omega$ by $\omega^{-1}$.

$$
0 \longrightarrow \omega^{-1} \longrightarrow E_{e} \longrightarrow \omega \longrightarrow 0
$$

Once chosen appropriate compatible linearizations on $\operatorname{Pic}^{1}(C)$ and $C$, we show that the linear system $\mathbb{P} H^{0}\left(\operatorname{Pic}^{1}(C), 3 \Theta\right)_{+}^{*}$ can be injected in $\mathbb{P} E x t^{1}\left(\omega, \omega^{-1}\right)$ and that we have the following theorem. 
Theorem 0.0.2 Let $C$ be a smooth genus 2 curve. The moduli space of strictly semistable involution invariant extension classes of $\omega$ by $\omega^{-1}$ is the Weddle surface $W \subset \mathbb{P} H^{0}\left(\text { Pic }^{1}(C), 3 \Theta\right)_{+}^{*}$ associated to $\operatorname{Pic}^{1}(C)$.

Moreover, let $\operatorname{Sec}(C) \subset\left|\omega^{3}\right|^{*}$ be the secant variety of the curve $C$ tricanonically embedded, we show that $W$ is the (everywhere tangent) intersection of $\operatorname{Sec}(C)$ with the hyperplane given by $\mathbb{P} H^{0}\left(\operatorname{Pic}^{1}(C), 3 \Theta\right)_{+}^{*}$.

Acknowledgments. It is a pleasure to thank my thesis advisor Christian Pauly, without whose insight and suggestions this paper couldn't have been written. I'm also very grateful to Bert Van Geemen for the influence he has had on my formation and the passion he has transmitted me.

\section{Theta characteristics and congruence subgroups of $S p(4, \mathbb{Z})$}

\subsection{Theta characteristics}

For much of the material in this section the reference is [Bea91]. Let $(A, H)$ be a principally polarized abelian variety (ppav for short) of dimension $g$. We will denote $A[2]$ the group of 2-torsion points and let

$$
\langle,\rangle: A[2] \times A[2] \rightarrow\{ \pm 1\}
$$

be the symplectic form induced by the principal polarization.

A theta characteristic of $A$ is a quadratic form $\kappa: A[2] \rightarrow\{ \pm 1\}$ associated to the symplectic form $\langle$, $\rangle$, i.e. a function on $A[2]$ verifying

$$
\kappa(x+y) \kappa(x) \kappa(y)=\langle x, y\rangle,
$$

for every $x, y \in A[2]$. We will denote the set of theta characteristics by $\vartheta(A)$. Let $x, y \in A[2]$ and $\kappa \in \vartheta(A)$. The $\mathbb{F}_{2}$-vector space $A[2]$ acts on $\vartheta(A)$ in the following way

$$
(x \cdot \kappa)(y)=\langle x, y\rangle \kappa(y)
$$

and $\vartheta(A)$ is an $A[2]$-torsor w.r.t. this action. Let $\kappa$ be an element of $\vartheta(A)$, there exists a number $\epsilon(\kappa) \in\{ \pm 1\}$ s.t. $\kappa$ takes the value $+\epsilon(\kappa)$ (resp. $-\epsilon(\kappa))$ at $2^{g-1}\left(2^{g}+1\right)$ points (resp. $2^{g-1}\left(2^{g}-1\right)$ points). The theta characteristic is said to be even if $\epsilon(\kappa)=+1$, odd in the opposite case, we will write $\vartheta^{+}(A)$ and $\vartheta^{-}(A)$ for the two sets just defined. Given $x \in A[2]$, $\epsilon$ satisfies

$$
\epsilon(x \cdot \kappa)=\kappa(x) \epsilon(\kappa) .
$$

Let $T(A)$ be the $A[2]$-torsor of symmetric theta divisors representing the polarization, there is a canonical identification of $A[2]$-torsors (which we will implicitly make in what follows) 


$$
\begin{aligned}
\vartheta(A) & \stackrel{\sim}{\longrightarrow} T(A), \\
\kappa & \mapsto \Theta_{\kappa} .
\end{aligned}
$$

This sends a theta characteristic $\kappa$ of $A$ to a symmetric theta divisor $\Theta_{\kappa}$ on $A$ characterized by the formula

$$
\kappa(a)=(-1)^{m_{a}\left(\Theta_{\kappa}\right)+m_{0}\left(\Theta_{\kappa}\right)},
$$

where $a \in A[2]$ and $m_{a}\left(\Theta_{\kappa}\right)$ is the multiplicity of the divisor $\Theta_{\kappa}$ at the point $a$. Let $a \in A$ and $t_{a}$ be the translation $x \mapsto x+a$ in $A$, then $\Theta_{a \cdot \kappa}=t_{a}^{*} \Theta_{\kappa}$ and

$$
\epsilon(\kappa)=(-1)^{m_{0}\left(\Theta_{\kappa}\right)} .
$$

Thus the fact that a theta characteristic is even or odd depends on the local equation of $\Theta_{\kappa}$ at the origin.

Remark 1.1.1 Suppose $A=J a c(C)$ is the Jacobian of a curve $C$, and denote by $\vartheta(C) \subset$ $P i c^{g-1}$ the set of the theta characteristics of $C$, i.e. line bundles $L$ s.t. $L^{2}=\omega$. Then $\vartheta(C) \cong$ $\vartheta(J a c(C)) \cong T(J a c(C))$ as $J a c(C)[2]$-torsors by $L \mapsto \Theta_{L}=\left\{M \in J a c(C) \mid H^{0}(L \otimes M) \neq 0\right\}$; and $\epsilon$ is the usual parity function by the Riemann singularity theorem.

Let us denote by $\imath$ the involution $-I d$ on the ppav $A$. Let $\theta_{\kappa}$ be a non zero section of $\mathcal{O}_{A}\left(\Theta_{\kappa}\right)$, and $\phi$ the unique isomorphism between $\imath^{*} \mathcal{O}_{A}\left(\Theta_{\kappa}\right)$ and $\mathcal{O}_{A}\left(\Theta_{\kappa}\right)$ which induces the identity over the origin. Following Mumford [Mum66], we will call $\phi$ the normalized isomorphism. Then we have

$$
\phi\left(\imath^{*} \theta_{\kappa}\right)=\epsilon(\kappa) \theta_{\kappa} .
$$

Definition 1.1.2 Let $L$ be a symmetric line bundle representing the polarization $H$, let $\phi$ : $L \rightarrow \imath^{*} L$ be the normalized isomorphism and $x \in A[2]$. We define $e_{*}^{L}(x)$ as the scalar $\alpha$ s.t.

$$
\phi(x): L_{x} \stackrel{\sim}{\rightarrow}\left(\imath^{*} L\right)_{x}=L_{\imath(x)}=L_{x}
$$

is the multiplication by $\alpha$.

The function that associates the scalar $e_{*}^{L}(x)$ to a point $x \in A[2]$ is a quadratic form on $A[2]$ and, if $\kappa \in \vartheta(A)$ then $e_{*}^{\mathcal{O}\left(\Theta_{\kappa}\right)}$ is the quadratic form $\kappa$ [Mum66]. We will often say that a line bundle is even (resp. odd) if the induced quadratic form on $A[2]$ is even (resp. odd).

Any given $\kappa \in \vartheta(A)$ can be used to identify $\vartheta(A)$ with $A[2]$, via the isomorphism

$$
\begin{aligned}
A[2] & \stackrel{\sim}{\longrightarrow} \vartheta(A), \\
x & \mapsto x \cdot \kappa .
\end{aligned}
$$




\subsection{Moduli spaces and subgroups of $S p(2 g, \mathbb{Z})$}

Let $g$ be a positive integer and $\Gamma_{g}=S p(2 g, \mathbb{Z})$ the full Siegel modular group of genus $g$. When necessary, we will use for the elements $M \in \Gamma_{g}$ the usual decomposition in four $g \times g$ blocks, $M=\left(\begin{array}{cc}A & B \\ C & D\end{array}\right)$ and if $Z$ is a square matrix, we will write $Z^{t}$ for its transpose. The group $\Gamma_{g}$ acts properly discontinuously and holomorphically on the Siegel upper half-plane

$$
\mathbb{H}_{g}:=\left\{\Omega \in \operatorname{Mat}_{g}(\mathbb{C}) \mid \Omega=\Omega^{t}, \operatorname{Im}(\Omega)>0\right\}
$$

by the formula

$$
M \cdot \Omega=(A \Omega+B)(C \Omega+D)^{-1} .
$$

The quotient $\mathcal{A}_{g}:=\mathbb{H}_{g} / \Gamma_{g}$ is a quasi-projective variety and it can be seen as the coarse moduli space of ppav of dimension $g$ [Igu72]. Let $m$ be a vector of $\left(\frac{1}{2} \mathbb{Z} / \mathbb{Z}\right)^{2 g}$. Such a vector is usually called a half-integer characteristic and we will call $a$ and $b$ the first and respectively the second $g$-coordinates of $m$. Once we choose a $\Omega \in \mathbb{H}_{g}$, we can associate to every halfinteger characteristic a holomorphic theta function on the abelian variety corresponding to $\Omega \bmod \Gamma_{g}$ as follows

$$
\Theta\left[\begin{array}{l}
a \\
b
\end{array}\right](\mathbf{z} ; \Omega):=\sum_{r \in \mathbb{Z}^{g}} e^{\pi i\left(\left(\mathbf{r}+\frac{1}{2} a\right) \cdot \Omega \cdot\left(\mathbf{r}+\frac{1}{2} a\right)+2\left(\mathbf{z}+\frac{1}{2} b\right) \cdot\left(\mathbf{r}+\frac{1}{2} a\right)\right)} .
$$

Moreover the zero divisor of $\Theta\left[\begin{array}{l}a \\ b\end{array}\right]$ is a symmetric theta divisor. Thus, via the identification 4, one can define (although non canonically) bijections between the set of half-integer characteristics and $\vartheta(A)$. Furthermore, the action of $\Gamma_{g}$ on $\Omega \in \mathbb{H}_{g}$ induces a transformation formula for theta functions with characteristics ([Igu64], Section 2). The induced action on the characteristics is then the following

$$
M \cdot\left(\begin{array}{c}
a \\
b
\end{array}\right)=\left(\begin{array}{cc}
D & -C \\
-B & A
\end{array}\right)\left(\begin{array}{c}
a \\
b
\end{array}\right)+\frac{1}{2}\left(\begin{array}{c}
\operatorname{diag}\left(C D^{t}\right) \\
\operatorname{diag}\left(A B^{t}\right)
\end{array}\right) .
$$

Lemma 1.2.1 ([Igu64], Section 2)

The action of $\Gamma_{g}$ on $\left(\frac{1}{2} \mathbb{Z} / \mathbb{Z}\right)^{2 g}$ defined by (8) has two orbits distinguished by the invariant

$$
\mathbf{e}(m)=(-1)^{4 a b^{t}} \in\{ \pm 1\} .
$$

We say that $m$ is an even (resp. odd) half-integer characteristic if $\mathbf{e}(m)=1$ (resp. $\mathbf{e}(m)=$ -1 ) and this invariant coincides via (4) with the invariant $\epsilon$ defined on theta characteristics in Section 1. Let us denote by

$$
\Gamma_{g}(3):=\operatorname{Ker}(S p(2 g, \mathbb{Z}) \rightarrow S p(2 g, \mathbb{Z} / 3 \mathbb{Z}))
$$


the principal congruence group of level 3 and by $\Gamma_{g}(3,6)$ the subgroup of $\Gamma_{g}(3)$ defined by $\operatorname{diag}\left(C D^{t}\right) \equiv \operatorname{diag}\left(A B^{t}\right) \equiv 0 \bmod 6$. The subgroup $\Gamma_{g}(3,6)$ then coincides with the stabilizer of the even theta characteristic $\left(\begin{array}{l}0 \\ 0\end{array}\right)$.

\section{Symmetric theta structures}

Let $(A, H)$ be a ppav of dimension $g$ and let $L$ be a symmetric line bundle that induces the polarization on it. Let $z \in A$ and $t_{z}$ be the translation $x \mapsto x+z$ on $A$. The level 3 (and genus g) theta group of $L$ is defined in the following way

$$
\mathcal{G}\left(L^{3}\right)=\left\{(\varphi, \eta) \mid \eta \in A, \varphi: t_{\eta}^{*}\left(L^{3}\right) \stackrel{\sim}{\rightarrow}\left(L^{3}\right)\right\},
$$

where the group law is $(\varphi, \eta) \cdot\left(\varphi^{\prime}, \eta^{\prime}\right)=\left(t_{\eta^{\prime}}^{*} \varphi \circ \varphi^{\prime}, \eta+\eta^{\prime}\right)$.

Group theoretically one can see $\mathcal{G}\left(L^{3}\right)$ as a central extension

$$
1 \longrightarrow \mathbb{C}^{*} \stackrel{i}{\longrightarrow} \mathcal{G}\left(L^{3}\right) \stackrel{p}{\longrightarrow} A[3] \longrightarrow 1,
$$

where the image of $\alpha$ via $i$ is the automorphism of $L^{3}$ given by the multiplication by $\alpha$ and $p(\varphi, \eta)=\eta$. The commutator $\left[(\varphi, \eta),\left(\varphi^{\prime}, \eta^{\prime}\right)\right]$ of two elements of $\mathcal{G}\left(L^{3}\right)$ belongs to the center of the group and it induces the Weil pairing

$$
e^{L}: A[3] \times A[3] \rightarrow \mathbb{C}^{*}
$$

taking lifts. Two different lifts give the same commutator.

As an abstract group $\mathcal{G}\left(L^{3}\right)$ is isomorphic to the Heisenberg group

$$
\mathcal{H}_{g}(3):=\mathbb{C}^{*} \times(\mathbb{Z} / 3 \mathbb{Z})^{g} \times(\widehat{\mathbb{Z} / 3 \mathbb{Z}})^{g},
$$

where $(\widehat{\mathbb{Z} / 3 \mathbb{Z}})^{g}:=\operatorname{Hom}\left((\mathbb{Z} / 3 \mathbb{Z})^{g}, \mathbb{C}^{*}\right)$. The group law in $\mathcal{H}_{g}(3)$ is not the product law but the following

$$
\left(t, x, x^{*}\right) \cdot\left(s, y, y^{*}\right)=\left(s t \omega^{y^{*}(x)}, x+y, x^{*}+y^{*}\right),
$$

where $\omega$ is a cubic root of 1 . The projection $\left(t, x, x^{*}\right) \mapsto\left(x, x^{*}\right)$ defines a central extension of groups

$$
1 \longrightarrow \mathbb{C}^{*} \longrightarrow \mathcal{H}_{g}(3) \longrightarrow(\mathbb{Z} / 3 \mathbb{Z})^{2 g} \longrightarrow 1 \text {. }
$$

Let $u:=\left(x, x^{*}\right), v:=\left(y, y^{*}\right) \in(\mathbb{Z} / 3 \mathbb{Z})^{2 g}$ and $\tilde{u}, \tilde{v} \in \mathcal{H}_{g}(3)$ two lifts. Then the commutator $[\tilde{u}, \tilde{v}]$ does not depend on the choice of the lifts and it defines the standard symplectic form $E$ on $(\mathbb{Z} / 3 \mathbb{Z})^{2 g}$, that is

$$
\begin{aligned}
E:(\mathbb{Z} / 3 \mathbb{Z})^{2 g} \times(\mathbb{Z} / 3 \mathbb{Z})^{2 g} & \longrightarrow \mathbb{C}^{*} ; \\
(u, v) & \mapsto \quad[\tilde{u}, \tilde{v}]=\omega^{x^{*}(y)-y^{*}(x)}
\end{aligned}
$$


A level 3 theta structure for $(A, L)$ is an isomorphism

$$
\alpha: \mathcal{H}_{g}(3) \stackrel{\sim}{\rightarrow} \mathcal{G}\left(L^{3}\right)
$$

which is the identity once restricted to $\mathbb{C}^{*}$.

Projecting on $(\mathbb{Z} / 3 \mathbb{Z})^{2 g}$, a level 3 theta structure $\alpha$ induces an isomorphism

$$
\tilde{\alpha}:(\mathbb{Z} / 3 \mathbb{Z})^{2 g} \stackrel{\sim}{\rightarrow} A[3]
$$

which is symplectic w.r.t. the Weil pairing on $A[3]$ and the standard symplectic pairing on $(\mathbb{Z} / 3 \mathbb{Z})^{2 g} \times(\mathbb{Z} / 3 \mathbb{Z})^{2 g}$. Such an isomorphism is called a level 3 structure on $(A, L)$.

Let $V_{3}(g)$ be the vector space of complex valued functions over $(\mathbb{Z} / 3 \mathbb{Z})^{g}$. It is well known, by the work of Mumford [Mum66], that a level 3 theta structure $\alpha$ induces an isomorphism (unique up to a scalar) between the $3^{g}$-dimensional vector spaces $H^{0}\left(A, L^{3}\right)$ and $V_{3}(g)$. This allows us to identify $\mathbb{P} H^{0}\left(A, L^{3}\right)$ with the abstract $\mathbb{P}^{3^{g}-1}=\mathbb{P}\left(V_{3}(g)\right)$ and to equip it with a canonical basis corresponding to the functions $\left\{X_{\alpha}\right\} \in \operatorname{Funct}\left((\mathbb{Z} / 3 \mathbb{Z})^{g}, \mathbb{C}\right)$, defined in the following way

$$
\begin{aligned}
X_{\alpha}:(\mathbb{Z} / 3 \mathbb{Z})^{g} & \longrightarrow \mathbb{C}, \\
X_{\alpha}(\alpha) & =1, \\
X_{\alpha}(\sigma) & =0 \text { if } \sigma \neq \alpha .
\end{aligned}
$$

There exists only one irreducible representation of $\mathcal{H}_{g}(3)$ on $V_{3}(g)$ where $\mathbb{C}^{*}$ acts linearly (this is usually called a level 1 representation): the so-called Schrödinger representation $U$. Let $\left(t, x, x^{*}\right)$ be an element of $\mathcal{H}_{g}(3)$ and $X_{\alpha} \in V_{3}(g)$, then

$$
U\left(t, x, x^{*}\right) \cdot X_{\alpha}=t x^{*}(\alpha+x) X_{\alpha+x} .
$$

Remark 2.0.2 Let $\mathcal{A}_{g}(3)$ be the moduli space of ppas with a level 3 structure and $\mathcal{A}_{g}(3,6)$ the moduli space of ppas with a level 3 structure and an even theta characteristic. The groups $\Gamma_{g}(3)$ and $\Gamma_{g}(3,6)$ defined in Section 1 act properly discontinuously and holomorphically on the Siegel upper half-plane $\mathbb{H}_{2}$ inducing the isomorphisms $\mathcal{A}_{g}(3) \cong \mathbb{H}_{g} / \Gamma_{g}(3)$ and $\mathcal{A}_{g}(3,6) \cong$ $\mathbb{H}_{g} / \Gamma_{g}(3,6)$.

\subsection{The action of $\imath$}

Let $(A, H)$ and $L$ be as in the preceding paragraph and $\phi: L \stackrel{\sim}{\rightarrow} \imath^{*} L$ be the normalized isomorphism. This isomorphism induces involutions $\imath^{\#}: H^{0}\left(A, L^{n}\right) \rightarrow H^{0}\left(A, L^{n}\right)$ for every $n$, defined in the following way

$$
\imath^{\#}(s)=\imath^{*}\left(\phi^{n}(s)\right)
$$


For our goals, it is useful to have an intrinsic computation of the dimensions of $H^{0}\left(A, L^{n}\right)_{+}$ et $H^{0}\left(A, L^{n}\right)_{-}$, that we will make by means of the Atiyah-Bott-Lefschetz fixed point formula ([GH78], p. 421). We know that the fixed points of $\imath$ are 2-torsion points, thus

$$
\sum_{j=0}^{2}(-1)^{j} \operatorname{Tr}\left(\imath^{\#}: H^{j}(A, L)\right)=\sum_{\beta \in A[2]} \frac{\operatorname{Tr}\left(\imath: L_{\beta} \rightarrow L_{\beta}\right)}{\operatorname{det}\left(I d-(d i)_{\beta}\right)} .
$$

Now $(d i)=-I d$ so $\operatorname{det}(2 I d)=2^{g}$. Recalling section 1.1, if the symmetric line bundle $L$ is even, we have

$$
\sum_{\beta \in A[2]} \operatorname{Tr}\left(\imath: L_{\beta} \rightarrow L_{\beta}\right)=2^{g-1}\left(2^{g}+1\right)-2^{g-1}\left(2^{g}-1\right)=2^{g},
$$

otherwise $-2^{g}$. Furthermore, as $L$ represents a principal polarization, $h^{p}(A, L)=0$ for $p>0$. Therefore, by definition of $H^{0}(A, L)_{+}$and $H^{0}(A, L)_{-}$,

$$
\sum_{j=0}^{2}(-1)^{j} \operatorname{Tr}\left(\imath^{\#}: H^{j}(A, L)\right)=h^{0}(A, L)_{+}-h^{0}(A, L)_{-} .
$$

Developing this formula we find that, for an even line bundle representing the polarization,

$$
\begin{gathered}
h^{0}(A, L)_{+}+h^{0}(A, L)_{-}=1 \\
h^{0}(A, L)_{+}-h^{0}(A, L)_{-}=1,
\end{gathered}
$$

which implies $h^{0}(L)_{+}=1$ and $h^{0}(L)_{-}=0$. If the line bundle is odd, we have

$$
\begin{gathered}
h^{0}(A, L)_{+}+h^{0}(A, L)_{-}=1 \\
h^{0}(A, L)_{+}-h^{0}(A, L)_{-}=-1,
\end{gathered}
$$

and the dimensions of the eigenspaces are respectively 0 and 1 .

If we are instead considering the $n$-th power of $L$ then the parity of $n$ comes into play, because $e_{*}^{L^{n}}(x)=e_{*}^{L}(x)^{n}$. Therefore, if $n \equiv 0 \bmod 2$, the parity of the line bundle is not important and we have

$$
\begin{aligned}
& h^{0}\left(A, L^{n}\right)_{+}+h^{0}\left(A, L^{n}\right)_{-}=n^{g} \\
& h^{0}\left(A, L^{n}\right)_{+}-h^{0}\left(A, L^{n}\right)_{-}=2^{g} .
\end{aligned}
$$

This implies $h^{0}\left(A, L^{n}\right)_{+}=\left(n^{g}+2^{g}\right) / 2$ and $h^{0}\left(A, L^{n}\right)_{-}=\left(n^{g}-2^{g}\right) / 2$. If $n \equiv 1 \bmod 2$ we need to make different calculations depending on the parity of the line bundle. These calculations, that we omit as they come from considerations very similar to the preceding ones, are summarized in the following Proposition (BL here means base locus). 
Proposition 2.1.1 Let $A$ be an abelian variety of dimension $g, n$ a positive integer and $L$ a symmetric line bundle on $A$ s.t. $h^{0}(A, L)=1$. The $2^{2 g}$ 2-torsion points are divided into two sets defined in the following way

$$
\begin{array}{r}
S_{+}:=\left\{x \in A[2] \text { s.t. } e_{*}^{L}(x)=1\right\}, \\
S_{-}:=\left\{x \in A[2] \text { s.t. } e_{*}^{L}(x)=-1\right\} .
\end{array}
$$

If $n$ is odd then, depending on the parity of $L$, we have:

\section{L even:}

1. $\#\left(S_{+}\right)=2^{g-1}\left(2^{g}+1\right)$ and $\#\left(S_{-}\right)=2^{g-1}\left(2^{g}-1\right)$;

2. $h^{0}\left(A, L^{n}\right)_{+}=\left(n^{g}+1\right) / 2$ and $h^{0}\left(A, L^{n}\right)_{-}=\left(n^{g}-1\right) / 2$.

\section{$L$ odd:}

1. $\#\left(S_{-}\right)=2^{g-1}\left(2^{g}+1\right)$ and $\#\left(S_{+}\right)=2^{g-1}\left(2^{g}-1\right)$;

2. $h^{0}\left(A, L^{n}\right)_{+}=\left(n^{g}-1\right) / 2$ and $h^{0}\left(A, L^{n}\right)_{-}=\left(n^{g}+1\right) / 2$.

In both cases $\boldsymbol{B} \boldsymbol{L}\left(\left|L^{n}\right|_{+}\right)=S_{-}, \boldsymbol{B} \boldsymbol{L}\left(\left|L^{n}\right|_{-}\right)=S_{+}$and the origin $0 \in S_{+}$. If $n$ is even, then

$$
h^{0}\left(A, L^{n}\right)_{+}=\left(n^{g}+2^{g}\right) / 2, \quad h^{0}\left(A, L^{n}\right)_{-}=\left(n^{g}-2^{g}\right) / 2 .
$$

Moreover $\left|L^{n}\right|_{+}$is base point free and $\boldsymbol{B} \boldsymbol{L}\left(\left|L^{n}\right|_{-}\right)=A[2]$.

Proof: We remark that for every positive integer $n$,

$$
\mathbf{B L}\left(\left|L^{n}\right|_{+}\right) \cup \mathbf{B L}\left(\left|L^{n}\right|_{-}\right)=A[2] .
$$

Let $n$ be odd. Since we use the linearization given by the normalized isomorphism, the assertion about the origin is true by definition. It remains to prove the assertion about the base locus. We recall that, if $x \in A[2], e_{*}^{L}(x)$ is the scalar $\alpha$ s.t. $\phi(x): L_{\imath(x)} \cong L_{x} \rightarrow L_{x}$ is the multiplication by $\alpha$. Thus, given an invariant section $\varphi \in H^{0}\left(A, L^{n}\right)_{+}$and $y \in S_{-}$, we have

$$
\varphi(y)=\left(\imath^{\#}(\varphi)\right)(y)=-\varphi(y),
$$

so $\varphi(y)=0$. This implies that all invariant sections must vanish at points of $S_{-}$. A similar argument shows that all anti-invariant sections vanish at points of $S_{+}$.

If $n$ is even, then we can write $n=2 k$ for some $k \in \mathbb{N}$. We recall that the linear system $\left|L^{2}\right|$ is base point free and that all sections of $H^{0}\left(A, L^{2}\right)$ are invariant. Then the linear system $\operatorname{Sym}^{k}\left(H^{0}\left(A, L^{2}\right)\right)$ is also base point free and by taking the restriction of $\operatorname{Sym}^{k}\left(H^{0}\left(A, L^{2}\right)\right)$ to 
$A$ we find a subspace of $H^{0}\left(A, L^{2 k}\right)+$ without base points. This implies that the whole linear system is base point free. We recall that, for $y \in A[2], e_{*}^{L^{2} k}(y)=e_{*}^{L}(y)^{2 k}$. Then $e_{*}^{L^{2} k}(z)=1$ for every $z \in A[2]$. This implies, by an argument similar to the one used for $n$ odd, that every $\varphi \in H^{0}\left(A, L^{2 k}\right)_{-}$must vanish at the 2 -torsion points.

A theta structure allows to take a canonical basis for $\mathbb{P} H^{0}\left(A, L^{3}\right)$. The rest of this section will be devoted to the study of the theta structures that define canonical bases also for the eigenspaces we have just described.

Definition 2.1.2 [Mum66] Let $\mathcal{G}\left(L^{3}\right)$ be the level 3 theta group and $\phi^{3}: L^{3} \stackrel{\sim}{\longrightarrow} \imath^{*} L^{3}$ the normalized isomorphism for $L^{3}$. Furthermore let $(x, \rho)$ be an element of $\mathcal{G}\left(L^{3}\right)$. We will denote by $\delta_{-1}: \mathcal{G}\left(L^{3}\right) \rightarrow \mathcal{G}\left(L^{3}\right)$ the automorphism of $\mathcal{G}\left(L^{3}\right)$ defined by taking the composition

$$
L \stackrel{\phi^{3}}{\longrightarrow} \imath^{*} L^{3} \stackrel{\imath^{*}(\rho)}{\longrightarrow} \imath^{*} t_{x}^{*} L^{3}=t_{-x}^{*} \imath^{*} L^{3} \stackrel{t_{-x}^{*} \phi^{3}}{\longleftarrow} t_{-x}^{*} L^{3}
$$

and setting

$$
\delta_{-1}((x, \rho)):=\left(-x,\left(t_{-x}^{*} \phi^{3}\right)^{-1} \circ\left(\imath^{*} \rho\right) \circ \phi^{3}\right) .
$$

Furthermore $\delta_{-1}$ decomposes in the following way

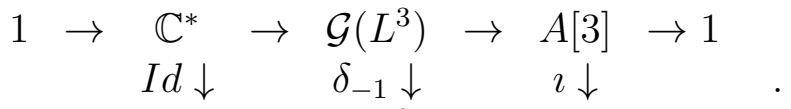

$$
\begin{aligned}
& 1 \rightarrow \mathbb{C}^{*} \rightarrow \mathcal{G}\left(L^{3}\right) \rightarrow A[3] \rightarrow 1
\end{aligned}
$$

Note that $\delta_{-1}$ is the only involution which lifts $\imath$ to $\mathcal{G}\left(L^{3}\right)$. This means that, if we denote by $\rho: \mathcal{G}\left(L^{3}\right) \rightarrow G L\left(H^{0}\left(A, L^{3}\right)\right)$ the natural representation of the theta group, the following diagram commutes for all $g \in \mathcal{G}\left(L^{3}\right)$ up to a scalar.

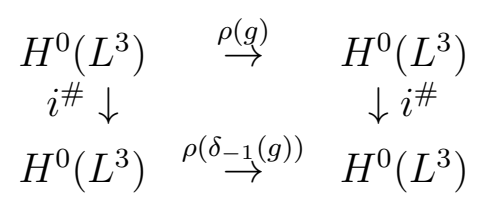

In the same way one can define an automorphism of the Heisenberg group

$$
\begin{aligned}
D_{-1}: \mathcal{H}_{g}(3) & \longrightarrow \mathcal{H}_{g}(3), \\
\left(t, x, x^{*}\right) & \mapsto\left(t,-x,-x^{*}\right) .
\end{aligned}
$$

In fact this automorphism makes the following diagram commute

$$
\begin{aligned}
& 1 \rightarrow \mathbb{C}^{*} \rightarrow \mathcal{H}_{g}(3) \rightarrow(\mathbb{Z} / 3 \mathbb{Z})^{g} \times(\mathbb{Z} / 3 \mathbb{Z})^{g} \rightarrow 1 \\
& I d \downarrow \quad D_{-1} \downarrow \quad-I d \downarrow \\
& 1 \rightarrow \mathbb{C}^{*} \rightarrow \mathcal{H}_{g}(3) \rightarrow(\mathbb{Z} / 3 \mathbb{Z})^{g} \times(\mathbb{Z} / 3 \mathbb{Z})^{g} \rightarrow 1 \text {. }
\end{aligned}
$$


Definition 2.1.3 Let Aut $\left(\mathcal{H}_{g}(3)\right)$ be the group of automorphisms of the Heisenberg group. We will denote

$$
A\left(\mathcal{H}_{g}(3)\right)=\left\{\phi \in \operatorname{Aut}\left(\mathcal{H}_{g}(3)\right): \phi((t, 0,0))=(t, 0,0), \forall t \in \mathbb{C}^{*}\right\} .
$$

Remark 2.1.4 If $\varphi \in A\left(\mathcal{H}_{g}(3)\right)$, then $U \circ \varphi$ is also a level 1 representation, thus by the Schur lemma there exists a unique linear map $T_{\varphi}: V_{3}(g) \rightarrow V_{3}(g)$, defined up to homothety, s.t. $T_{\varphi}(U(h))=U(\varphi(h))$ for all $h \in \mathcal{H}_{g}(3)$. In this way we obtain a projective representation

$$
\begin{aligned}
\widetilde{T}: A\left(\mathcal{H}_{g}(3)\right) & \longrightarrow \mathbb{P} G L\left(V_{3}(g)\right), \\
\varphi & \mapsto T_{\varphi} \bmod \mathbb{C}^{*} .
\end{aligned}
$$

Let $X_{\sigma}:(\mathbb{Z} / 3 \mathbb{Z})^{g} \rightarrow \mathbb{C}$ be the canonical basis of $V_{3}(g)$ s.t. $X_{\sigma}(\sigma)=1, X_{\sigma}(\alpha)=0$ if $\alpha \neq \sigma \in(\mathbb{Z} / 3 \mathbb{Z})^{g}$. We note that $D_{-1} \in A\left(\mathcal{H}_{g}(3)\right)$. Then a lift $j$ in $G L\left(V_{3}(g)\right)$ of $\widetilde{T}\left(D_{-1}\right)$ is given as follows

$$
\begin{aligned}
j: V_{3}(g) & \longrightarrow V_{3}(g) \\
X_{\sigma} & \mapsto X_{-\sigma} .
\end{aligned}
$$

Note that the lift of $\widetilde{T}\left(D_{-1}\right)$ is only defined up to \pm 1 . Furthermore $j$ makes the following diagram commute, for every $h \in \mathcal{H}_{g}(3)$

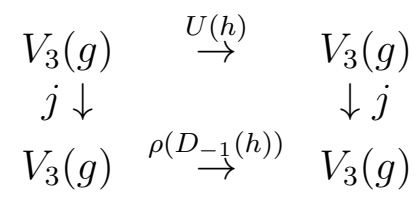

This action decomposes $V_{3}(g)$ into a direct sum of two eigenspaces $V_{3}(g)_{+} \oplus V_{3}(g)_{-}$. We are now ready to define the theta structure we need.

Definition 2.1.5 A level 3 theta structure $\alpha: \mathcal{G}\left(L^{3}\right) \rightarrow \mathcal{H}_{g}(3)$ is said to be symmetric if the following diagram commutes

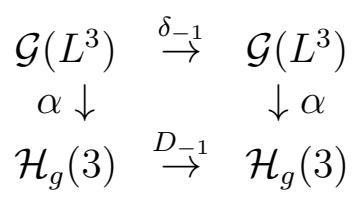

Such a theta structure allows us to take a canonical basis not only for $H^{0}\left(A, L^{3}\right)$ but also for $H^{0}\left(A, L^{3}\right)_{+}$and $H^{0}\left(A, L^{3}\right)_{-}$. 


\subsection{Automorphisms of the Heisenberg group}

Let $n$ be an odd positive integer, $n \neq 1$. Two different level $n$ theta structures differ by an element of $A\left(\mathcal{H}_{g}(n)\right)$. Furthermore we have the following Proposition.

Proposition 2.2.1 The group $A\left(\mathcal{H}_{g}(n)\right)$ fits into the following exact sequence

$$
1 \rightarrow(\mathbb{Z} / n \mathbb{Z})^{2 g} \rightarrow A\left(\mathcal{H}_{g}(n)\right) \rightarrow S p(2 g, \mathbb{Z} / n \mathbb{Z}) \rightarrow 1 .
$$

Proof: First of all we define the homomorphisms. Let $u:=\left(x, x^{*}\right), v:=\left(y, y^{*}\right) \in(\mathbb{Z} / n \mathbb{Z})^{2 g}$. For $\phi \in A\left(\mathcal{H}_{g}(n)\right)$ and $\left(t, x, x^{*}\right) \in \mathcal{H}_{g}(n)$, we have

$$
\phi\left(t, x, x^{*}\right)=\phi(t, 0,0) \phi\left(1, x, x^{*}\right)=(t, 0,0) \phi\left(1, x, x^{*}\right) .
$$

Thus we can write

$$
\phi\left(t, x, x^{*}\right)=\left(f_{\phi}\left(x, x^{*}\right) t, G_{\phi}\left(x, x^{*}\right)\right)
$$

for an automorphism $G_{\phi}:(\mathbb{Z} / n \mathbb{Z})^{2 g} \rightarrow(\mathbb{Z} / n \mathbb{Z})^{2 g}$ and a function $f_{\phi}:(\mathbb{Z} / n \mathbb{Z})^{2 g} \rightarrow \mathbb{C}^{*}$. Moreover the map

$$
\begin{aligned}
G: A\left(\mathcal{H}_{g}(n)\right) & \longrightarrow \operatorname{Aut}\left((\mathbb{Z} / n \mathbb{Z})^{2 g}\right) \\
\phi & \mapsto G_{\phi}
\end{aligned}
$$

is a homomorphism. Consider $\phi \in \operatorname{ker}(G)$. Then $f_{\phi}$ is a group homomorphism since $\phi$ is an automorphism. All such homomorphism are of the form

$$
f_{\phi}\left(t, x, x^{*}\right)=\omega^{E(a, u)} \text { for some } a \in(\mathbb{Z} / n \mathbb{Z})^{2 g},
$$

where $E(-,-)$ is the standard symplectic $\mathbb{Z} / n \mathbb{Z}$-valued form on $(\mathbb{Z} / n \mathbb{Z})^{2 g} \times(\mathbb{Z} / n \mathbb{Z})^{2 g}$ and $\omega^{n}=1$. So we obtain a homomorphism

$$
\begin{aligned}
\zeta:(\mathbb{Z} / n \mathbb{Z})^{2 g} & \longrightarrow A\left(\mathcal{H}_{g}(n)\right) \\
a & \mapsto\left[\left(t, x, x^{*}\right) \stackrel{\zeta_{a}}{\mapsto}\left(t \omega^{E(u, a)}, x, x^{*}\right)\right]
\end{aligned}
$$

The homomorphisms $\zeta$ and $G$ are respectively the first and the second arrow in the sequence 14. Moreover, since $\phi \in A\left(\mathcal{H}_{g}(n)\right)$, it preserves the commutators. This means that

$$
\begin{aligned}
& \omega^{E(u, v)}=\quad[\tilde{u}, \tilde{v}]=\quad \tilde{u} \cdot \tilde{v} \cdot \tilde{u}^{-1} \cdot \tilde{v}^{-1}= \\
& =\phi\left(\tilde{u} \cdot \tilde{v} \cdot \tilde{u}^{-1} \cdot \tilde{v}^{-1}\right)=[\phi(\tilde{u}), \phi(\tilde{v})]=\omega^{E\left(G_{\phi}(u), G_{\phi}(v)\right)},
\end{aligned}
$$

and thus $\operatorname{Im}(G) \subset S p(2 g, \mathbb{Z} / n \mathbb{Z})$. It is also easy to see that $\zeta$ is injective and that $G \circ$ $\zeta\left(\left(x, x^{*}\right)\right)=I d, \forall\left(x, x^{*}\right) \in(\mathbb{Z} / n \mathbb{Z})^{2 g}$.

The subgroup of $A\left(\mathcal{H}_{g}(n)\right)$ of elements of the form $\alpha \circ \beta^{-1}$, with $\alpha, \beta$ symmetric theta structures, is easily identified with the centralizer of $D_{-1}$, denoted by $C_{A\left(\mathcal{H}_{g}(n)\right)}\left(D_{-1}\right)$. In order to finish the proof of Proposition 2.2.1 we need the following Lemma. 
Lemma 2.2.2 The homomorphism $G$ induces an isomorphism

$$
G: C_{A\left(\mathcal{H}_{g}(n)\right)}\left(D_{-1}\right) \stackrel{\sim}{\longrightarrow} S p(2 g, \mathbb{Z} / n \mathbb{Z})
$$

Proof: Let $\zeta$ be the first map of the sequence 14 . Then, for all $a \in(\mathbb{Z} / n \mathbb{Z})^{2 g}$

$$
\begin{gathered}
D_{-1} \circ \zeta_{a}\left(t, x, x^{*}\right)=\left(t \omega^{E(u, a)},-x,-x^{*}\right), \\
\zeta_{a} \circ D_{-1}\left(t, x, x^{*}\right)=\left(t \omega^{E(-u, a)},-x,-x^{*}\right) .
\end{gathered}
$$

Thus $D_{-1} \circ \zeta_{a}(t, x)=\zeta_{a} \circ D_{-1}(t, x)$ if and only if $-E(x, a)=E(x, a)$. This is impossible, as it would imply $E(x, a)=0$ for all $a$ and $E$ is non-degenerate. This implies that $\operatorname{Im}(\zeta) \cap$ $C_{A\left(\mathcal{H}_{g}(n)\right)}\left(D_{-1}\right)=I d$, i.e. $G_{\mid C_{A\left(\mathcal{H}_{g}(n)\right)}\left(D_{-1}\right)}$ is injective.

Let $M \in S p(2 g, \mathbb{Z} / n \mathbb{Z})$, now we show that there exists a lift $\widetilde{M} \in C_{A\left(\mathcal{H}_{g}(n)\right)}\left(D_{-1}\right) \subset A\left(\mathcal{H}_{g}(n)\right)$ over M, i.e. $G_{\widetilde{M}}=M$.

The problem reduces to find a function $f_{M}:(\mathbb{Z} / n \mathbb{Z})^{2 g} \rightarrow \mathbb{C}$ s.t. there exists an automorphism $\widetilde{M} \in C_{A\left(\mathcal{H}_{g}(n)\right)}\left(D_{-1}\right)$ of the form

$$
\widetilde{M}\left(t, x, x^{*}\right)=\left(f_{M}\left(x, x^{*}\right) t, M\left(x, x^{*}\right)\right) .
$$

The fact that $\widetilde{M} \in C_{A\left(\mathcal{H}_{g}(n)\right)}\left(D_{-1}\right)$ implies that $f_{M}\left(-x,-x^{*}\right)=f_{M}\left(x, x^{*}\right)$. Furthermore we need $\widetilde{M}$ to be an automorphism of $\mathcal{H}_{g}(n)$. This means that for all $\left(x, x^{*}\right),\left(y, y^{*}\right) \in(\mathbb{Z} / n \mathbb{Z})^{2 g}$, if we denote by $\left(a, a^{*}\right)$ (resp. $\left.\left(b, b^{*}\right)\right)$ the image $M\left(x, x^{*}\right)$ (resp. the image $\left.M\left(y, y^{*}\right)\right), f_{M}$ should satisfy the equation

$$
f_{M}\left(x+y, x^{*}+y^{*}\right) \omega^{y^{*}(x)}=f_{M}\left(x, x^{*}\right) \cdot f_{M}\left(y, y^{*}\right) \omega^{b^{*}(a)},
$$

in order to have $\widetilde{M}\left(\left(t, x, x^{*}\right) \cdot\left(s, y, y^{*}\right)\right)=\widetilde{M}\left(t, x, x^{*}\right) \cdot \widetilde{M}\left(s, y, y^{*}\right)$. Let $\beta$ be the bilinear form

$$
\begin{aligned}
\beta:(\mathbb{Z} / n \mathbb{Z})^{2 g} \times(\mathbb{Z} / n \mathbb{Z})^{2 g} & \longrightarrow \mathbb{Z} / n \mathbb{Z} \\
\left(\left(x, x^{*}\right),\left(y, y^{*}\right)\right) & \mapsto y^{*}(x) .
\end{aligned}
$$

Its relation with the standard symplectic form $E$ on $(\mathbb{Z} / n \mathbb{Z})^{2 g}$ is given by the formula

$$
E\left(\left(x, x^{*}\right),\left(y, y^{*}\right)\right)=y^{*}(x)-x^{*}(y)=\beta\left(\left(x, x^{*}\right),\left(y, y^{*}\right)\right)-\beta\left(\left(y, y^{*}\right),\left(x, x^{*}\right)\right) .
$$

We assume that $f_{M}$ is of the form $f_{M}=\omega^{\phi_{M}}$ for some function $\phi_{M}:(\mathbb{Z} / n \mathbb{Z})^{2 g} \rightarrow(\mathbb{Z} / n \mathbb{Z})$. Then equation (16) is equivalent to the equation

$$
\begin{array}{r}
\phi_{M}\left(x+y, x^{*}+y^{*}\right)-\phi_{M}\left(x, x^{*}\right)-\phi_{M}\left(y, y^{*}\right)=b^{*}(a)-y^{*}(x)= \\
=\beta\left(M\left(x, x^{*}\right), M\left(y, y^{*}\right)\right)-\beta\left(\left(x, x^{*}\right),\left(y, y^{*}\right)\right) .
\end{array}
$$

Now we observe that the function 


$$
\begin{aligned}
\psi:(\mathbb{Z} / n \mathbb{Z})^{2 g} \times(\mathbb{Z} / n \mathbb{Z})^{2 g} & \longrightarrow \mathbb{Z} / n \mathbb{Z} \\
\left(\left(x, x^{*}\right),\left(y, y^{*}\right)\right) & \mapsto \beta\left(M\left(x, x^{*}\right), M\left(y, y^{*}\right)\right)-\beta\left(\left(x, x^{*}\right),\left(y, y^{*}\right)\right)
\end{aligned}
$$

is symmetric. In fact, for all $a=\left(x, x^{\prime}\right), b=\left(y, y^{\prime}\right) \in(\mathbb{Z} / n \mathbb{Z})^{2 g}$

$$
\begin{gathered}
\psi(a, b)-\psi(b, a)=\beta(M(a), M(b))-\beta(a, b)-\beta(M(b), M(a))+\beta(b, a)= \\
=E(M(a), M(b))-E(a, b)=0,
\end{gathered}
$$

since $M \in S p(2 g, \mathbb{Z} / n \mathbb{Z})$.

Let $a:=\left(a_{1}, a_{2}\right) \in(\mathbb{Z} / n \mathbb{Z})^{2 g}$ and let $\phi_{M}$ be the quadratic form associated to the symmetric bilinear form $\psi$, i.e.

$$
\begin{aligned}
\phi_{M}:(\mathbb{Z} / n \mathbb{Z})^{2 g} & \longrightarrow \mathbb{C} \\
\left(a_{1}, a_{2}\right) & \mapsto \frac{1}{2}\left[\beta\left(M\left(a_{1}, a_{2}\right), M\left(a_{1}, a_{2}\right)\right)-\beta\left(\left(a_{1}, a_{2}\right)\right)\right]
\end{aligned}
$$

Then the polarization formula gives equation 17 and

$$
\begin{aligned}
\widetilde{M}: \mathcal{H}_{g}(n) & \longrightarrow \mathcal{H}_{g}(n) \\
\left(t, x, x^{*}\right) & \mapsto\left(t \omega^{\phi_{M}\left(x, x^{*}\right)}, M\left(x, x^{*}\right)\right)
\end{aligned}
$$

is an automorphism of $\mathcal{H}_{g}(n)$. Moreover $\omega^{\phi_{M}\left(x, x^{*}\right)}=\omega^{\phi_{M}\left(-x,-x^{*}\right)}$ so $\widetilde{M} \in C_{A\left(\mathcal{H}_{g}(n)\right)}\left(D_{-1}\right)$ and $\widetilde{M}$ is a lift over $M$. This ends the Proof of Lemma 2.2.2.

Corollary 2.2.3 Let $(A, H)$ be a ppav and $L$ a line bundle representing the polarization. Then a level 3 structure determines a unique symmetric theta structure of level $n$.

Lemma 2.2.2 implies that the second arrow of the sequence 14 is surjective, thus completing the Proof of Proposition 2.2.1.

Corollary 2.2.4 For every odd integer $n$ there is an isomorphism

$$
A\left(\mathcal{H}_{g}(n)\right) \cong S p(2 g, \mathbb{Z} / n \mathbb{Z}) \ltimes(\mathbb{Z} / n \mathbb{Z})^{2 g},
$$

where the action of $S p(2 g, \mathbb{Z} / n \mathbb{Z})$ on $(\mathbb{Z} / n \mathbb{Z})^{2 g}$ is that induced by $G L(2 g, \mathbb{Z} / n \mathbb{Z})$ on $(\mathbb{Z} / n \mathbb{Z})^{2 g}$.

Proof: We note that, since it is the kernel of the homomorphism $G,(\mathbb{Z} / n \mathbb{Z})^{2 g}$ is a normal subgroup of $A\left(\mathcal{H}_{g}(n)\right)$. Then $S p(2 g, \mathbb{Z} / n \mathbb{Z}) \cong C_{A\left(\mathcal{H}_{g}(n)\right)}\left(D_{-1}\right)$ acts on $(\mathbb{Z} / n \mathbb{Z})^{2 g}$ by conjugation. Let $a=\left(a_{1}, a_{2}\right) \in(\mathbb{Z} / n \mathbb{Z})^{2 g}$ and let $\zeta_{a} \in A\left(\mathcal{H}_{g}(n)\right)$ the automorphism defined in (15). Moreover let $\widetilde{M} \in A\left(\mathcal{H}_{g}(n)\right)$ the lift of $M \in S p(2 g, \mathbb{Z} / n \mathbb{Z})$ defined in the proof of Lemma 2.2.2. Then, for all $a \in(\mathbb{Z} / n \mathbb{Z})^{2 g}$, we have 


$$
\begin{aligned}
J: S p(2 g, \mathbb{Z} / n \mathbb{Z}) & \longrightarrow G L\left((\mathbb{Z} / n \mathbb{Z})^{2 g}\right. \\
M & \mapsto J_{M}:=\left[\zeta_{a} \mapsto \widetilde{M} \circ \zeta_{a} \circ \widetilde{M}^{-1}\right] .
\end{aligned}
$$

An easy calculation shows that

$$
\widetilde{M} \circ \zeta_{a} \circ \widetilde{M}^{-1}\left(t, x, x^{*}\right)=\zeta_{M \cdot a}\left(t, x, x^{*}\right),
$$

where $M \cdot a$ is the natural action of $S p(2 g, \mathbb{Z} / n \mathbb{Z})$ on $(\mathbb{Z} / n \mathbb{Z})^{2 g}$

Furthermore the inclusion of $S p(2 g, \mathbb{Z} / n \mathbb{Z})$ in $A\left(\mathcal{H}_{g}(n)\right)$ as the subgroup $C_{A\left(\mathcal{H}_{g}(n)\right)}\left(D_{-1}\right)$ gives a representation

$$
\Upsilon: S p(2 g, \mathbb{Z} / n \mathbb{Z}) \longrightarrow \mathbb{P} G L\left(V_{n}(g)\right)
$$

by restriction of the representation $\widetilde{T}$ defined in (12) for level 3. Moreover, since $S p(2 g, \mathbb{Z} / n \mathbb{Z}) \cong$ $C_{A\left(\mathcal{H}_{g}(n)\right)}\left(D_{-1}\right)$, the representation $\Upsilon$ decomposes in two subrepresentations

$$
\begin{aligned}
& \Upsilon_{+}: S p(2 g, \mathbb{Z} / n \mathbb{Z}) / \pm I d \longrightarrow \mathbb{P} G L\left(V_{n}(g)\right)_{+}, \\
& \Upsilon_{-}: S p(2 g, \mathbb{Z} / n \mathbb{Z}) / \pm I d \longrightarrow \mathbb{P} G L\left(V_{n}(g)\right)_{-} .
\end{aligned}
$$

\section{The arithmetic group $\Gamma_{2}(3)^{-}$}

Lemma 3.0.5 We have the exact sequence

$$
1 \longrightarrow \Gamma_{2}(6) \longrightarrow \Gamma_{2}(3) \stackrel{\text { mod }}{\longrightarrow} S p(4, \mathbb{Z} / 2 \mathbb{Z}) \longrightarrow 1 .
$$

Proof:The first arrow is the natural inclusion. To prove the surjectivity of the second one we need the following formula given by Igusa ([Igu64], page 222)

$$
\left[\Gamma_{g}: \Gamma_{g}(n)\right]=n^{g(2 g+1)} \prod_{p \mid n} \prod_{1 \leq k \leq g}\left(1-p^{-2 k}\right) .
$$

This gives the following indexes

$$
\begin{array}{lcc}
{\left[\Gamma_{2}: \Gamma_{2}(3)\right]=} & 51840 & =\# S p(4, \mathbb{Z} / 3 \mathbb{Z}) ; \\
{\left[\Gamma_{2}: \Gamma_{2}(2)\right]=} & 720 & =\# S p(4, \mathbb{Z} / 2 \mathbb{Z}) ; \\
{\left[\Gamma_{2}: \Gamma_{2}(6)\right]=} & 720 \times 51840 & =\# S p(4, \mathbb{Z} / 6 \mathbb{Z}),
\end{array}
$$

and the fact that $\left[\Gamma_{2}: \Gamma_{2}(6)\right] /\left[\Gamma_{2}: \Gamma_{2}(3)\right]=\# S p(4, \mathbb{Z} / 2 \mathbb{Z})$ implies the surjectivity of the second arrow. 
In the section 1.2 we have seen that the action of $\Gamma_{g}$ on characteristics has two orbits and that, by equation 8 , the group $\Gamma_{g}(3,6) \subset \Gamma_{g}(3)$ could be seen by definition as the stabilizer subgroup in $\Gamma_{g}(3)$ of the even characteristic $\left(\begin{array}{l}0 \\ 0\end{array}\right)$.

Let $(V,\langle\rangle$,$) be a 4$-dimensional symplectic vector space over $\mathbb{Z} / 2 \mathbb{Z}$ and let $Q V$ denote the set of all quadratic forms on $V$, relative to $\langle$,$\rangle . There are 16$ quadratic forms in $Q V$ and they divide into two sets of 10 and 6 elements distinguished by the Arf invariant. When we are considering theta characteristics this invariant coincides with the parity defined in section 1.1. Furthermore $Q V$ is a principal homogeneous space for $V$, which endows the disjoint union $Z=V \cup Q V$ with the structure of a $\mathbb{Z} / 2 \mathbb{Z}$-vector space of dimension 5 .For a more complete exposition about quadratic forms on $\mathbb{Z} / 2 \mathbb{Z}$-vector spaces we refer e.g. to [GH04].

Recalling the bijection between half-integer characteristics and theta characteristics one sees that $\Gamma_{2}(3,6)$ fits in the following exact sequence

$$
1 \longrightarrow \Gamma_{2}(6) \longrightarrow \Gamma_{2}(3,6) \stackrel{\text { mod } 2}{\longrightarrow} O^{+}(4, \mathbb{Z} / 2 \mathbb{Z}) \longrightarrow 1,
$$

where $O^{+}(4, \mathbb{Z} / 2 \mathbb{Z}) \subset S p(4, \mathbb{Z} / 2 \mathbb{Z})$ is the stabilizer subgroup of an even quadratic form on $(\mathbb{Z} / 2 \mathbb{Z})^{4}$. The group we are interested in is the odd analogue of $\Gamma_{2}(3,6)$. Let $O^{-}(4, \mathbb{Z} / 2 \mathbb{Z}) \subset$ $S p(4, \mathbb{Z} / 2 \mathbb{Z})$ be the stabilizer subgroup of an odd quadratic form.

Proposition 3.0.6 [GH04]

We have an isomorphism

$$
\operatorname{Sp}(4, \mathbb{Z} / 2 \mathbb{Z}) \cong \Sigma_{6}
$$

under which $S p(4, \mathbb{Z} / 2 \mathbb{Z})$ acts on the set of odd quadratic forms by permutation. Furthermore, let $\tilde{q}$ be an odd quadratic form, then

$$
O(4, \tilde{q}) \cong O^{-}(4, \mathbb{Z} / 2 \mathbb{Z}) \cong \Sigma_{5} \subset \Sigma_{6}
$$

Definition 3.0.7 We will denote by $\Gamma_{2}(3)^{-}$the group that fits in the following exact sequence

$$
1 \longrightarrow \Gamma_{2}(6) \longrightarrow \Gamma_{2}(3)^{-} \stackrel{\text { mod } 2}{\longrightarrow} O^{-}(4, \mathbb{Z} / 2 \mathbb{Z}) \longrightarrow 1 \text {. }
$$

Then we have $\Gamma_{2}(6) \subset \Gamma_{2}(3)^{-} \subset \Gamma_{2}(3)$ and $\left[\Gamma_{2}(3): \Gamma_{2}(3)^{-}\right]=6$.

Let

$$
M: O(4, \mathbb{Z} / 2 \mathbb{Z})^{-} \longrightarrow S p(4, \mathbb{Z} / 2 \mathbb{Z}) \subset G L\left((\mathbb{Z} / 2 \mathbb{Z})^{4}\right) .
$$

be the natural inclusion, then we have the following Theorem. 
Theorem 3.0.8 As an arithmetic group, $\Gamma_{2}(3)^{-}$is the group of matrices $G=\left(\begin{array}{cc}A & B \\ C & D\end{array}\right) \in$ $S p(4, \mathbb{Z})$ such that $G \equiv I_{4} \bmod 3, G \equiv M(\sigma) \bmod 2$, for some $\sigma \in O\left(4, \mathbb{F}_{2}\right)^{-}$.

Let $\mathcal{A}_{2}(3)^{-}$be the fine moduli space parametrizing the triples $(A, L, \theta)$, where $A$ is an abelian surface, $L$ is a symmetric ample odd line bundle s.t. $h^{0}(A, L)=1$ and $\theta$ is a symmetric theta structure of level 3.

Corollary 3.0.9 The quasi-projective variety $\mathbb{H}_{2} / \Gamma_{2}(3)^{-}$is the fine moduli space $\mathcal{A}_{2}(3)^{-}$of ppas with a symmetric level 3 theta structure and an odd theta characteristic.

Proof: We only have to prove that the quotient $\mathbb{H}_{2} / \Gamma_{2}(3)^{-}$is a fine moduli space, but $\Gamma_{2}(3)^{-} \subset \Gamma_{2}(3)$ and $\Gamma_{2}(3)$ are torsion free and this implies the assertion.

\section{The moduli space $\mathcal{A}_{2}(3)^{-}$and Weddle surfaces}

\subsection{The Burkhardt quartic and the moduli space $\mathcal{A}_{2}(3)^{-}$}

Let $(A, H, \theta)$ be an irreducible ppas with a level 3 theta structure and $L$ a symmetric line bundle representing $H$. Let $\varphi_{L^{3}}(A) \subset \mathbb{P} H^{0}\left(A, L^{3}\right)^{*}$ be the image of $A$ given by $3^{\text {rd }}$ order theta functions. The theta structure gives an identification $\Phi_{\theta}: \mathbb{P} H^{0}\left(A, L^{3}\right)^{*} \cong \mathbb{P} V_{3}(2)^{*}=\mathbb{P}^{8}$ so that we can look at the image $\Phi_{\theta}\left(\varphi_{L^{3}}(A)\right) \subset \mathbb{P} V_{3}(2)^{*}$. From now on we will often denote $\Phi\left(\varphi_{L^{3}}(A)\right)$ simply by $A$. Let $\left\{X_{\sigma}\right\}_{\sigma \in(\mathbb{Z} / 3 \mathbb{Z})^{2}}$ be the basis of $V_{3}(2)$ given in (11). We introduce the two lagrangian subgroups of $\mathcal{H}_{2}(3)$ :

$$
\begin{aligned}
K & =\left\{\left(t, x, x^{*}\right): t=1, x=0\right\} \\
K^{*} & =\left\{\left(t, x, x^{*}\right): t=1, x^{*}=0\right\} .
\end{aligned}
$$

Note that $\mathbb{C}^{*}, K$ and $K^{*}$ generate $\mathcal{H}_{2}(3)$ and that $K$ acts by scalar multiplication on the basis $X_{\sigma}$ of $V_{3}(2)$, whereas $K^{*}$ acts by permuting these basis elements. The vector space of quadrics $H^{0}\left(\mathbb{P}^{8}, \mathcal{I}_{A}(2)\right)$ which are identically zero on $A$ is 9-dimensional. Moreover we underline the fact that $H^{0}\left(\mathbb{P}^{8}, \mathcal{O}_{\mathbb{P}^{8}}(2)\right)$ is a $\mathcal{H}_{2}(3)$-module and $H^{0}\left(\mathbb{P}^{8}, \mathcal{I}_{A}(2)\right)$ is an irreducible subrepresentation. Van der Geer ([vdG87], Section 1 and 2) remarked that each such subrepresentation contains a $K$-invariant quadric. Such quadrics span a 5-dimensional vector space $\operatorname{Sym}^{2} V_{3}(2)^{K} \subset \operatorname{Sym}^{2} V_{3}(2)$ and a basis of $\operatorname{Sym}^{2} V_{3}(2)^{K}$ is given by the binomials $X_{\alpha} X_{-\alpha}$, for $\alpha \in(\mathbb{Z} / 3 \mathbb{Z})^{2}$. Thus we have a $f_{0} \in H^{0}\left(\mathbb{P}^{8}, \mathcal{I}_{\varphi_{A}}(2)\right)$ of the form - up to a scalar -

$$
f_{0}=\sum_{\sigma} r_{\sigma} X_{\sigma} X_{-\sigma}
$$

with $r_{\sigma}=r_{-\sigma}$. Then by letting $K^{*} \cong(\mathbb{Z} / 3 \mathbb{Z})^{2}$ act on $f_{0}$, we have that

$$
a \cdot f_{0}=f_{a}=\sum_{\sigma} r_{\sigma} X_{\sigma+a} X_{-\sigma+a}, \quad a \in(\mathbb{Z} / 3 \mathbb{Z})^{2},
$$


give a complete basis for $H^{0}\left(\mathbb{P}^{8}, \mathcal{I}_{\varphi_{L^{3}}(A)}(2)\right)$.

Let us suppose now that our ppas has a level 3 symmetric theta structure. Then we can take canonical bases for the eigenspaces of $V_{3}(2)^{*}$ w.r.t. the action of the involution $j$ defined in equation 13 . We introduce the new coordinates

$$
\begin{aligned}
& Y_{\sigma}=\left(X_{\sigma}+X_{-\sigma}\right) / 2, \\
& Z_{\sigma}=\left(X_{\sigma}-X_{-\sigma}\right) / 2, \quad \sigma \neq 0 .
\end{aligned}
$$

The $Y_{\sigma}$ provide coordinates for $V_{3}(2)_{+}^{*}$, while the $Z_{\sigma}$ for $V_{3}(2)_{-}^{*}$. We will denote by $\mathbb{P}_{-}^{3}$ the projectivized space $\mathbb{P} V_{3}(2)_{-}^{*}$ and by $\mathbb{P}_{+}^{4}$ the projectivized $\mathbb{P} V_{3}(2)_{+}^{*}$. Moreover if $L$ is even (resp. odd) we have an identification of $\left|L^{3}\right|_{+}$with $\mathbb{P}_{+}^{4}$ (resp. $\mathbb{P}_{-}^{3}$ ). We have instead an identification of $\left|L^{3}\right|_{-}$with $\mathbb{P}_{-}^{3}$ (resp. $\mathbb{P}_{+}^{4}$ ) if $L$ is even (resp. odd). Then, recalling Proposition 2.1.1, we have

$$
\begin{aligned}
& A \cap \mathbb{P}_{-}^{3}=S_{+} \text {if } \mathrm{L} \text { is odd, } S_{-} \text {if } \mathrm{L} \text { is even, } \\
& A \cap \mathbb{P}_{+}^{4}=S_{-} \text {if } \mathrm{L} \text { is odd, } S_{+} \text {if } \mathrm{L} \text { is even. }
\end{aligned}
$$

Let $\operatorname{Hess}(\mathcal{B}) \subset \mathbb{P}_{+}^{4}$ be the Hessian hypersurface of the Burkhardt quartic. Van der Geer showed that the Theta-null map $T h^{+}$induces a birational isomorphism

$$
\begin{aligned}
T h^{+}: \mathcal{A}_{2}(3)^{+} & \stackrel{\sim}{\longrightarrow} \operatorname{Hess}(\mathcal{B}) \\
(A, L, \vartheta) & \mapsto \Phi_{\vartheta}\left(\varphi_{L^{3}}(0)\right)
\end{aligned}
$$

where 0 is the origin of the ppas and $\Phi_{\theta}$ is the identification of $\mathbb{P}_{+}^{4}$ with $\mathbb{P} H^{0}\left(A, L^{3}\right)_{+}^{*}$ given by the symmetric theta structure $\vartheta$. In fact once we restrict the quadrics of $H^{0}\left(\mathbb{P}^{8}, \mathcal{I}_{A}(2)\right)$ to $\mathbb{P}^{4}$ we obtain five quadrics $Q_{i}\left[\ldots: Y_{\sigma}: \ldots\right]$, for $i=1, \ldots, 5$. We can write the $Q_{i}$ down as a matrix $M_{+}\left[Y_{i}\right]$ with quadratic entries that multiplies the vector of the coefficients.

$$
\left(\begin{array}{c}
Q_{1} \\
Q_{2} \\
Q_{3} \\
Q_{4} \\
Q_{5}
\end{array}\right):=\left(\begin{array}{ccccc}
Y_{0}^{2} & Y_{1}^{2} & Y_{2}^{2} & Y_{3}^{2} & Y_{4}^{2} \\
Y_{1}^{2} & Y_{0} Y_{1} & Y_{3} Y_{4} & Y_{2} Y_{4} & Y_{2} Y_{3} \\
Y_{2}^{2} & Y_{3} Y_{4} & Y_{0} Y_{2} & Y_{1} Y_{4} & Y_{3} Y_{1} \\
Y_{3}^{2} & Y_{2} Y_{4} & Y_{1} Y_{4} & Y_{0} Y_{3} & Y_{1} Y_{2} \\
Y_{4}^{2} & Y_{3} Y_{2} & Y_{1} Y_{3} & Y_{1} Y_{2} & Y_{0} Y_{4}
\end{array}\right)\left(\begin{array}{c}
r_{0} \\
r_{1} \\
r_{2} \\
r_{3} \\
r_{4}
\end{array}\right)=0
$$

and the image of $T h^{+}$is the locus where this matrix has positive corank. Furthermore $M_{+}\left[Y_{i}\right]$ is the Hessian matrix of $\mathcal{B}$ and thus we have the isomorphism of (24).

We remark that the vector space $S y m^{2} V_{3}(2)^{K}$ can be identified with $V_{3}(2)_{+}$in the following way. 


$$
\begin{aligned}
\operatorname{Sym}^{2} V_{3}(2)^{K} & \stackrel{\sim}{\longrightarrow} V_{3}(2)_{+} \\
\sum_{i} a_{i} X_{\alpha} X_{-\alpha} & \mapsto \sum_{i} a_{i} Y_{\alpha}
\end{aligned}
$$

Then we have the natural 10:1 Steinerian map

$$
\begin{aligned}
S t_{+}: \operatorname{Hess}(\mathcal{B}) & \longrightarrow \mathbb{P S y m}{ }^{2} V_{3}(2)^{K} \cong \mathbb{P}_{+}^{4} \\
{\left[\ldots: b_{i}: \ldots\right] } & \mapsto \operatorname{Ker}\left(M_{+}\left[b_{i}\right]\right) .
\end{aligned}
$$

In fact, let $\left[\ldots: b_{i}: \ldots\right]$ be the coordinates of $T h^{+}(A, L, \vartheta)$, then $\operatorname{Ker}\left(M_{+}\left[b_{i}\right]\right)$ is the vector of the coefficients $r_{i}$ of the quadrics of $H^{0}\left(\mathbb{P}^{8}, \mathcal{I}_{A}(2)\right)$. The image of $S t_{+}$is called the Steinerian variety of $\mathcal{B}$ and it is denoted by $S t(\mathcal{B})$. Moreover, Hunt [Hun96] has proved that $S t(\mathcal{B}) \cong \mathcal{B}$, so that we have a 10:1 birational map $S t_{+}: \operatorname{Hess}(\mathcal{B}) \rightarrow \mathcal{B}$. Furthermore, the coefficients $r_{i}$ do not depend on the choice of the even symmetric line bundle $L$ in the triple $(A, L, \vartheta) \in \mathcal{A}_{2}(3)^{+}$, so that $S t_{+}$as a map is birational to the forgetful morphism $f$ that forgets the line bundle. This proves that there exists a birational isomorphism $Q: \mathcal{B} \stackrel{\sim}{\rightarrow} \mathcal{A}_{2}(3)$ and that we have the following commutative diagram.

$$
\begin{array}{ccc}
\mathcal{A}_{2}(3)^{+} & \stackrel{T h^{+}}{\longrightarrow} & \operatorname{Hess}(\mathcal{B}) \subset \mathbb{P}^{4} \\
f^{+} \downarrow & & \downarrow S t_{+} \\
\mathcal{A}_{2}(3) & \stackrel{Q}{\longrightarrow} & S t_{+}(\mathcal{B})=\mathcal{B}
\end{array}
$$

Let us look at the restriction to $\mathbb{P}_{-}^{3}$ of the linear system $\left|\mathcal{I}_{A}(2)\right|$. By writing the quadrics obtained as in (25), we have the following matrix equation.

$$
\left(\begin{array}{c}
Q_{1}^{\prime} \\
Q_{2}^{\prime} \\
Q_{3}^{\prime} \\
Q_{4}^{\prime} \\
Q_{5}^{\prime}
\end{array}\right):=\left(\begin{array}{ccccc}
0 & -Z_{1}^{2} & -Z_{2}^{2} & -Z_{3}^{2} & -Z_{4}^{2} \\
Z_{1}^{2} & 0 & -2 Z_{3} Z_{4} & -2 Z_{2} Z_{4} & -2 Z_{2} Z_{3} \\
Z_{2}^{2} & 2 Z_{3} Z_{4} & 0 & 2 Z_{1} Z_{4} & -2 Z_{3} Z_{1} \\
Z_{3}^{2} & 2 Z_{2} Z_{4} & -2 Z_{1} Z_{4} & 0 & 2 Z_{1} Z_{2} \\
Z_{4}^{2} & 2 Z_{3} Z_{2} & 2 Z_{1} Z_{3} & -2 Z_{1} Z_{2} & 0
\end{array}\right)\left(\begin{array}{c}
r_{0} \\
r_{1} \\
r_{2} \\
r_{3} \\
r_{4}
\end{array}\right)=0
$$

Let us denote by $M_{-}\left[Z_{i}\right]$ the skew-symmetric matrix of equation 27 . The determinant of $M_{-}\left[Z_{i}\right]$ is identically zero on $\mathbb{P}_{-}^{3}$. This allows us to define a Steinerian map

$$
\begin{aligned}
S t_{-}: \mathbb{P}_{-}^{3} & \longrightarrow \mathcal{B} \\
{\left[\ldots: s_{i}: \ldots\right] } & \mapsto \operatorname{Ker}\left(M_{-}\left[b_{i}\right]\right) .
\end{aligned}
$$

The matrix $M_{-}\left[Z_{i}\right]$ has rank 4 for a general $z=\left[\ldots: Z_{i}: \ldots\right] \in \mathbb{P}_{-}^{3}$. Then its comatrix has rang 1 and can be written as $\operatorname{Ker}\left(M_{-}\left[b_{i}\right]\right) \cdot \operatorname{Ker}\left(M_{-}\left[b_{i}\right]\right)^{t}$. This implies that $S t_{-}$is given by the system of quartics obtained as pfaffians of the skew-symmetric $4 \times 4$ minors obtained 
deleting the $j^{\text {th }}$ line and the $j^{\text {th }}$ column of the matrix, $j=1, \ldots, 5$. This gives the following quartics

$$
\begin{aligned}
& r_{0}=6 Z_{1} Z_{2} Z_{3} Z_{4}, \\
& r_{1}=Z_{1}\left(Z_{2}^{3}+Z_{3}^{3}-Z_{4}^{3}\right), \\
& r_{2}=-Z_{2}\left(Z_{1}^{3}+Z_{3}^{3}+Z_{4}^{3}\right), \\
& r_{3}=Z_{3}\left(-Z_{1}^{3}-Z_{2}^{3}+Z_{4}^{3}\right), \\
& r_{4}=Z_{4}\left(Z_{1}^{3}+Z_{2}^{3}-Z_{3}^{3}\right),
\end{aligned}
$$

that have 40 base points [Hun96].

Proposition 4.1.1 The Pfaffian construction gives the 6:1 unirationalization

$$
S t_{-}: \mathbb{P}_{-}^{3} \stackrel{\mathcal{O}(4)}{\longrightarrow} \mathcal{B}, \quad\left[\ldots: Z_{i}: \ldots\right] \mapsto\left[\ldots: r_{i}: \ldots\right],
$$

given by the system (28).

This calculation was given by Coble too [Cob17] via a different argument.

Lemma 4.1.2 By construction the fiber of St_ over a point $p \in \mathcal{B}$ are the six points (22) of the abelian surface whose ideal of quadrics is determined by $p$.

We recall from [Hun96] that there is a Zariski open subset of $\mathcal{B}$ which is biregular to a Zariski open subset of the moduli space $\mathcal{A}_{2}(3)$. The set contained in $\mathcal{A}_{2}(3)$ is exactly the set of irreducible abelian surfaces while the one in $\mathcal{B}$ is complementary to a system of 40 planes contained in $\mathcal{B}$. We will denote by $V^{\prime}$ the open set of $\mathcal{A}_{2}(3)$, by $V$ that of $\mathcal{B}$ and by $Q$ the morphism between them. For more details see [Hun96].

We are now ready to prove our first main result.

Proof of Theorem 0.0.1: Let $(A, L, \vartheta)$ be an element of $\mathcal{A}_{2}(3)^{-}$. We recall from Proposition that, when $L$ is odd, we have an identification $\Phi_{\vartheta}: \mathbb{P}_{-}^{3} \cong\left|L^{3}\right|_{+}$so that we have a theta-null map

$$
\begin{aligned}
T h^{-}: \mathcal{A}_{2}(3)^{-} & \longrightarrow \mathbb{P}_{-}^{3} \\
(A, L, \vartheta) & \mapsto \Phi_{\vartheta}\left(\varphi_{L^{3}}(0)\right) .
\end{aligned}
$$

$\mathcal{A}_{2}(3)^{-}$is a quotient of $\mathbb{H}_{2}$ by the arithmetic congruence group $\Gamma_{2}(3)^{-}$and so, by the BailyBorel Theorem [BB66], it is a quasi-projective variety. Let us consider the open set $U \subset$ $\mathcal{A}_{2}(3)^{-}$given by irreducible surfaces. Note that for surfaces in our moduli space, $0 \in S_{+}$ and $\mathbf{B L}\left|L^{3}\right|_{+}=S_{-}$. Thus the Theta-null map $T h^{-}$is everywhere defined and holomorphic on $U$. Let $(A, H, \varphi)$ be an irreducible ppas with level 3 structure and let 


$$
f^{-}: \mathcal{A}_{2}(3)^{-} \stackrel{6: 1}{\longrightarrow} \mathcal{A}_{2}(3)
$$

be the forgetful morphism that forgets the choice of the odd line bundle representing the polarization. The degree is 6 because of Corollary 2.2.3. The six objects in the fiber of $f^{-}$over $(A, H, \varphi)$ are sent via $T h^{-}$to the 6 points of $S_{+}$that are the intersection 22 . We remark that these six points constitute also the fiber via $S t_{-}$of the point of $B$ representing $(A, H, \varphi)$ and, as $(A, H, \varphi)$ moves in $V^{\prime}$, they cover the whole $\mathbb{P}_{+}^{3}$ because the determinant of the matrix of (27) is zero. Then we have the following commutative diagram of birational maps.

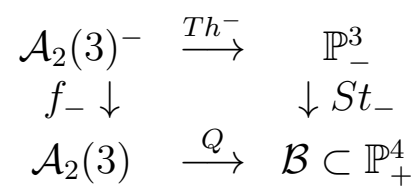

We note that this means that $T h^{-}$is a generically one to one map; as we are working in characteristic zero this implies the result. $\square$

Corollary 4.1.3 $\mathcal{A}_{2}(3)^{-}$is rational.

Remark 4.1.4 Let $p \in \mathbb{P}_{-}^{3}$, it is natural to ask whether it is possible to recover the triple $(A, L, \vartheta) \in \mathcal{A}_{2}(3)^{-}$s.t. $T h^{-}(A, L, \vartheta)=p$. The answer is positive. The coordinates in $\mathbb{P}_{+}^{4} \cong \mathbb{P} S y m^{2} V_{3}(2)^{K}$ of $S t_{-}(p)$ are the coefficients of the nine quadrics that vanish on $A \subset$ $\mathbb{P}^{8}=\mathbb{P} V_{3}(2)^{*}$. This gives us $A$. We remark that the action of $\mathcal{H}_{2}(3)$ gives an inclusion

$$
\rho:(\mathbb{Z} / 3 \mathbb{Z})^{4} \hookrightarrow P G L\left(V_{3}(2)\right)
$$

By taking the images of $0 \in A$ under the different projective transformations given by $\rho$ we obtain a level 3 structure. Moreover, if we consider the six points $S t_{-}^{*}\left(S t_{-}(p)\right)$, there exists a unique twisted cubic $R_{A}$ through them. Then the abelian variety $A$ can be seen as the Jacobian variety of the curve $X_{A}$ obtained as a covering of $R_{A} \cong \mathbb{P}^{1}$ branched in $S t_{-}^{*}\left(S t_{-}(p)\right)$. Now $p$ is a Weierstrass point of $X_{A}$, which is equivalent to an odd theta characteristic.

Remark 4.1.5 Salvati Manni and Freitag ([FSM04], Section 6) showed that the composition St_oTh-gives five functions $B_{1}, \ldots, B_{5}$ on $\mathbb{H}_{2}$ that are modular forms w.r.t. $\Gamma_{2}(3)$, thus giving another proof of the fact that $\mathcal{B}$ is birational to the Satake compactification of $\mathcal{A}_{2}(3)$. We conjecture that the the four components of Th- should be modular forms w.r.t. $\Gamma_{2}(3)^{-}$. Some related work has also been done by Ramanan and Adler [AR96].

\subsection{Weddle surfaces, Kummer surfaces and level 6 theta structures}

Let $\alpha:=(A, H, \varphi)$ be a ppas with a level 3 structure and $L$ a symmetric line bundle representing $H$. Once we have chosen $L$, the level 3 structure determines uniquely a symmetric level 3 theta structure and the image of $A$ in the space we called $\mathbb{P}_{-}^{3}$ in section 4.1 is a quartic 
surface $W_{\alpha}$ with six double points at six 2-torsion points. These points are in fact the fiber of $\pi$ over the point $p_{\alpha}$ of $\mathcal{B}$ representing the triple $(A, H, \varphi)$. This surface is commonly known as Weddle surface and as a projective variety it doesn't depend on the choice of the line bundle representing the polarization, but only on the level 3 structure. So, in some sense, $\mathcal{B}$ is a moduli space of Weddle surfaces. Coble made this statement clearer.

Lemma 4.2.1 [Cob17] The image $S t_{-}\left(W_{\alpha}\right)$ of the Weddle surface is the tangent hyperplane section of $\mathcal{B}$ at $p_{\alpha} \in \mathcal{B}$.

This surface was also known to classical geometers as it is the Jacobian surface associated to a Kummer symmetroid. It is in fact always possible to write the equation of a Kummer quartic surface as the determinant of a $4 \times 4$ symmetric matrix with linear entries (such a surface is called a symmetroid). Then to each point of the Kummer surface one can associate a degenerate quadratic form on a four dimensional vector space $V$. The Jacobian surface is then the locus in $\mathbb{P}(V)$ of the kernels of the degenerate matrices parametrized by the symmetroid and, in the case of the Kummer surface, its Jacobian surface is a Weddle surface.

It is possible to explain this in terms of spaces of theta functions. In the rest of this subsection we suppose that we have chosen a symmetric odd line bundle $L$. Then the Weddle surface $W$ is the image of the abelian variety $A$ in $\mathbb{P} H^{0}\left(A, L^{3}\right)_{+}^{*} \cong \mathbb{P}^{3}$.

Proposition 4.2.2 Let $(A, H)$ be a ppas and $L$ a symmetric odd line bundle representing $H$. There is a canonical injection (unique up to homothety)

$$
Q: H^{0}\left(A, L^{2}\right)^{*} \hookrightarrow \operatorname{Sym}^{2} H^{0}\left(A, L^{3}\right)_{+}
$$

whose image is the space of quadrics in $\left|L^{3}\right|_{+}^{*}$ passing through $S_{+} \subset A$.

Proof: Let $K$ be the Kummer surface contained in $\left|L^{2}\right|^{*}$ as the image of $A$. There exists a unique quartic $F \in S y m^{4} H^{0}\left(A, L^{2}\right)$ vanishing on $K$ invariant w.r.t. the action of $\mathcal{G}\left(L^{2}\right)$ on $\left|L^{2}\right|$. To each point $p$ of $\mathbb{P} H^{0}\left(A, L^{2}\right)^{*}$ we associate the polar cubic $\mathcal{P}_{p}(F)$ of $F$ with respect to $p$, thus identifying $H^{0}\left(A, L^{2}\right)^{*}$ with the 4-dimensional space $\mathcal{P}(F) \subset \operatorname{Sym}^{3} H^{0}\left(A, L^{2}\right)$ of polar cubics of $F$. We remark that for both $S_{y m^{2}} H^{0}\left(A, L^{3}\right)_{+}$and $\mathcal{P}(F)$ we have the injective evaluation maps

$$
\begin{aligned}
\mu_{1}: \operatorname{Sym}^{2} H^{0}\left(A, L^{3}\right)_{+} & \longrightarrow H^{0}\left(A, L^{6}\right)_{+}, \\
\mu_{2}: \mathcal{P}(F) \subset \operatorname{Sym}^{3} H^{0}\left(A, L^{2}\right) & \longrightarrow H^{0}\left(A, L^{6}\right)_{+} .
\end{aligned}
$$

Note that for dimensional reasons $H^{0}\left(A, L^{6}\right)_{+} \cong S y m^{3} H^{0}\left(A, L^{2}\right)$ via $\mu_{2}$. Moreover, recalling that $\mathbf{B L}\left|L^{3}\right|_{+}=S_{-}$(Prop. 2.1.1), we remark that $\mu_{1}\left(S y m^{2} H^{0}\left(A, L^{3}\right)_{+}\right)$is identified with the subspace of $\operatorname{Sym}^{3} H^{0}\left(A, L^{2}\right)$ given by cubics vanishing at $S_{-}$(because of dimensions). Furthermore $\mathcal{P}(F)$ is the subspace of cubics vanishing at $S_{+} \cup S_{-}=A[2]$. $Q$ is the unique (up to homothety) map that makes the following diagram commute. 


$$
\begin{aligned}
& H^{0}\left(A, L^{2}\right)^{*} \stackrel{\mathcal{P}(F)}{\hookrightarrow} \operatorname{Sym}^{3} H^{0}\left(A, L^{2}\right) \cong H^{0}\left(A, L^{6}\right)_{+} \\
& Q \searrow \quad \mu_{1} \uparrow \\
& \operatorname{Sym}^{2} H^{0}\left(A, L^{3}\right)_{+}
\end{aligned}
$$

This proves the Proposition. $\square$

In the following the index over a linear system indicates we are considering the subsystem with such a base locus. We then have the following classical Proposition.

Proposition 4.2.3 ([Har95], Theorem 22.33) Let $\mathcal{D}_{1}$ be the universal determinantal variety in $\mathbb{P}^{9}=\mathbb{P} S y m^{2} H^{0}\left(A, L^{3}\right)_{+}$and $\mathcal{D}_{2}$ its singular locus. Let $q \in \mathcal{D}_{1}-\mathcal{D}_{2}$ and $v \in \mathbb{P}_{+}^{3}$ its vertex; then

$$
T_{q} \mathcal{D}_{1}=\{\text { quadrics passing through } v\} .
$$

Furthermore $\mathcal{D}_{1}$ is singular in codimension 2 and the degree of its singular locus is 10 [HT84]. Let $\left\{v_{1}, \ldots, v_{6}\right\} \in \mathbb{P} H^{0}\left(A, L^{3}\right)_{+}^{*}$ be the points of $S_{+}$. Let $i=1, \ldots, 6$, there exists only one quadric of rank 3 in $\mathbb{P} H^{0}\left(A, L^{3}\right)_{+}^{*}$ having $v_{i}$ as vertex and vanishing at $S_{+}$. Let us call this quadric $q_{i}$. The six quadrics $q_{i}$ contain the unique twisted cubic vanishing on $S_{+}$and, by Proposition 4.2.3, we have that

$$
\mathbb{P} \operatorname{Sym}^{2} H^{0}\left(A, L^{3}\right)_{+}^{S_{+}}=\bigcap_{i=1}^{6} T_{q_{i}} \mathcal{D}_{1} .
$$

This means that the linear system of quadrics $\operatorname{Sym}^{2} H^{0}\left(A, L^{3}\right)_{+}^{S_{+}} \cong H^{0}\left(A, L^{2}\right)$ cuts out a quartic surface $S=\mathcal{D}_{1} \cap \mathbb{P} H^{0}\left(A, L^{2}\right)^{*} \subset \mathbb{P} S y m^{2} H^{0}\left(A, L^{3}\right)_{+}$that has 10 nodes given by the intersection $\mathcal{D}_{2} \cap \mathbb{P} H^{0}\left(A, L^{2}\right)^{*}$ plus 6 nodes at the quadrics $q_{i}$. Hudson [Hud90] also remarked that the 10 rank 2 quadrics of $\mathcal{D}_{2} \cap \mathbb{P} H^{0}\left(A, L^{2}\right)^{*}$ are defined in the following way. We take two complementary triples in $\left\{v_{1}, \ldots, v_{6}\right\}$ and each of them defines a 2-plane in $\mathbb{P} H^{0}\left(A, L^{3}\right)_{+}^{*}$. We have ten choices of this kind and each of the ten quadrics is the union of the two 2-planes defined by such a choice.

We are able to prove (but we will not go through the proof here as it is not very instructive) the following Proposition.

Proposition 4.2.4 The injection $Q$ identifies the Kummer surface $K \subset \mathbb{P}\left(H^{0}\left(A, L^{2}\right)^{*}\right.$ with the determinantal surface $S \subset \mathbb{P} S y m^{2} H^{0}\left(A, L^{3}\right)_{+}^{S_{+}}$.

The projective configuration of the Kummer and Weddle surfaces is summarized in the following diagram. Here $\pi_{S_{-}}$means the projection from $S_{-}$. The equalities on the right are to be intended once one evaluates everything in $H^{0}\left(A, L^{6}\right)_{+}$. 


$$
\begin{array}{cccc}
\mathbb{P}^{3} \supset K & \stackrel{\text { Ver }_{3}}{\longrightarrow} & \mathbb{P}\left(\text { Sym }^{3} H^{0}\left(A, L^{2}\right)\right) & =H^{0}\left(A, L^{6}\right)_{+} \\
\downarrow \pi_{S_{-}} & \\
\mathbb{P}^{3} \supset W & \stackrel{\text { Ver }}{\longrightarrow} & \mathbb{P}\left(S y m^{2} H^{0}\left(A, L^{3}\right)_{+}\right) & =\mathbb{P}\left(\operatorname{Sym}^{3} H^{0}\left(A, L^{2}\right)^{S_{-}}\right. \\
& & \downarrow \pi_{S_{+}} & \\
\mathbb{P}^{3} \supset K & \stackrel{\text { Polar }}{\longrightarrow} & \mathbb{P}\left(\text { Sym }^{2} H^{0}\left(A, L^{3}\right)_{+}^{S_{+}}\right) & =\mathbb{P}(\mathcal{P}(K))
\end{array}
$$

Remark 4.2.5 We also made some Riemann-Roch calculations on $\widetilde{K}$, the blow up of $K$ in its 16 nodes. We found a linear series of divisors, defined by the formula

$$
2 D \equiv 3 H-\sum_{p \in S_{-}} E_{p}
$$

where $H$ is the pull-back to $\widetilde{K}$ of a hyperplane section of $K$ and $E_{z} \cong \mathbb{P}^{1}$ is the exceptional divisor over the point $z \in K$. Easy calculations imply that $\operatorname{dim}|D|=3$ and $D^{2}=4$, exactly what we expected for the Weddle surface. Furthermore let $r_{i}$, for $i \in\{1, \ldots, 6\}$, be the points of $S_{+}$, then for all $i$ we have

$$
E_{r_{i}} \cdot\left(3 H-\sum_{p \in S_{-}} E_{p}\right)=0 .
$$

This means that the divisor $E_{r_{1}}+\cdots+E_{r_{6}}$ is, following Saint-Donat [SD74], the fundamental cycle of the linear system 31 and that $\widetilde{K}$ is isomorphic to the blow-up of $W$ in its six double points.

\section{$5 \quad$ Involution invariant vector bundles}

Let $C$ be a smooth curve of genus 2 and $\lambda$ the hyperelliptic involution on $C$; let also $\operatorname{Pic}{ }^{d}(C)$ be the Picard variety parametrizing degree $d$ line bundles over $C$ and $\operatorname{Jac}(C)=\operatorname{Pic} c^{0}(C)$ the Jacobian variety of $C$. We will denote $K^{0}$ the Kummer surface obtained as quotient of $\operatorname{Jac}(C)$ by $\pm I d$ and $K^{1}$ the quotient of $\operatorname{Pic}^{1}(C)$ by the involution $\tau: \xi \mapsto \omega \otimes \xi^{-1}$. Moreover we remark that the 16 theta characteristics are the fixed points of the involution $\tau$. Let $\Theta \subset \operatorname{Pic}^{1}(C)$ be the Riemann theta divisor and $\Theta_{0} \subset J a c(C)$ be a symmetric theta divisor, i.e. a translate of $\Theta$ by a theta-characteristic. We also recall that the two linear systems $|2 \Theta|$ and $\left|2 \Theta_{0}\right|$ are dual to each other via Wirtinger duality ([Mum74], p. 335), i.e. we have an isomorphism $|2 \Theta|^{*} \cong\left|2 \Theta_{0}\right|$. Let $\mathcal{M}_{C}$ be the moduli space of semi-stable rank two vector bundles on $C$ with trivial determinant. It is isomorphic to $\mathbb{P}^{3} \cong|2 \Theta|$, the isomorphism being given by the map [Bea88] 


$$
\begin{aligned}
\Delta: \mathcal{M}_{C} & \longrightarrow|2 \Theta|, \\
E & \mapsto \Delta(E) ;
\end{aligned}
$$

where

$$
\Delta(E):=\left\{L \in \operatorname{Pic}^{1}(C) \mid h^{0}(C, E \otimes L) \neq 0\right\} .
$$

With its natural scheme structure, $\Delta(E)$ is in fact linearly equivalent to $2 \Theta$. The Kummer surface $K^{0}$ is embedded in $|2 \Theta|$ and points in $K^{0}$ correspond to bundles $E$ whose Sequivalence class $[E]$ contains a decomposable bundle of the form $M \oplus M^{-1}$, for $M \in J a c(C)$. Furthermore on the semistable boundary the morphism $\Delta$ restricts to the Kummer map. The Riemann theta divisor $\Theta$ is invariant w.r.t. the involution $\tau$. This means that we have two possible choices for a linearization of the action of $\tau$ on $\mathcal{O}_{P_{i c}(C)}(\Theta)$ and the only section $\theta$ of $\mathcal{O}_{P_{i c^{1}}(C)}(\Theta)$ will be invariant or anti-invariant depending on the chosen linearization. We choose once and for all the linearization

$$
\nu: \tau^{*} \mathcal{O}_{P i c^{1}(C)}(\Theta) \stackrel{\sim}{\longrightarrow} \mathcal{O}_{P i c^{1}(C)}(\Theta)
$$

with respect to which $\theta \in H^{0}\left(\operatorname{Pic}^{1}(C), \Theta\right)_{-}$. By the Atyiah-Bott-Lefschetz fixed point formula [GH78] this means that $\nu$ induces $I d$ on the fiber of $\mathcal{O}_{P i c^{1}(C)}(\Theta)$ over each of the 6 odd theta characteristics and $-I d$ on the fiber over each of the 10 even theta characteristics. Always by the Atyiah-Bott-Lefschetz formula we find that this choice implies that

$$
\begin{gathered}
h^{0}\left(\operatorname{Pic}^{1}(C), 3 \Theta\right)_{-}=5, \\
h^{0}\left(\operatorname{Pic}^{1}(C), 3 \Theta\right)_{+}=4 .
\end{gathered}
$$

Remark 5.0.6 Let $\kappa \in P i c^{1}(C)$ be an odd theta characteristic and $\Theta_{0} \cong t_{\kappa}^{*} \Theta$ the symmetric theta divisor on $J a c(C)$ translate of $\Theta$ by $\kappa$. Then the linearization $\nu$ induces the normalized isomorphism

$$
\mathcal{O}_{J a c(C)}\left(\Theta_{0}\right) \stackrel{t_{\kappa}^{*} \nu}{\longrightarrow} t_{\kappa}^{*} \lambda^{*} \mathcal{O}_{P i c^{1}(C)}(\Theta) \cong \imath^{*} \mathcal{O}_{\text {Jac }(C)}\left(\Theta_{0}\right)
$$

for the symmetric line bundle $\mathcal{O}_{J a c(C)}\left(\Theta_{0}\right)$ on $\operatorname{Jac}(C)$. The quadratic form induced by $\mathcal{O}_{J a c(C)}\left(\Theta_{0}\right)$ on $J a c(C)$ is odd. We recall from proposition 2.1.1 that, for any odd positive integer $n$, this means that the base points of $H^{0}\left(J a c(C), n \Theta_{0}\right)_{ \pm}$are the subsets of $J a c(C)[2]$ where $\kappa$ takes the value $\mp 1$. Translating again by $\kappa$ and using equation 3 we find that

$$
\begin{aligned}
& \mathbf{B L}\left(|n \Theta|_{+}\right)=\text {even theta characteristics, } \\
& \mathbf{B L}\left(|n \Theta|_{-}\right)=\text {odd theta characteristics. }
\end{aligned}
$$




\subsection{Extensions of the canonical bundle}

Let $\omega$ be the canonical line bundle on $C$. We introduce the 4-dimensional projective space

$$
\mathbb{P}_{\omega}^{4}:=\mathbb{P} E x t^{1}\left(\omega, \omega^{-1}\right)=\left|\omega^{3}\right|^{*} .
$$

A point $e \in \mathbb{P}_{\omega}^{4}$ corresponds to an isomorphism class of extensions

$$
0 \longrightarrow \omega^{-1} \longrightarrow E_{e} \longrightarrow \omega \longrightarrow 0 .
$$

We denote by $\varphi$ the classifying map

$$
\begin{aligned}
\varphi: \mathbb{P}_{\omega}^{4} & \rightarrow|2 \Theta| \\
e & \mapsto \text { S-equivalence class of } E_{e} .
\end{aligned}
$$

Let $\mathcal{I}_{C}$ be the ideal sheaf of the curve $C \subset \mathbb{P}_{\omega}^{4}$, Bertram ([Ber92], Theorem 2) showed that there is an isomorphism (induced via pull-back by $\varphi$ )

$$
H^{0}\left(\mathcal{M}_{C}, \mathcal{O}(2 \Theta)\right) \cong H^{0}\left(\mathbb{P}_{\omega}^{4}, \mathcal{I}_{C} \otimes \mathcal{O}(2)\right) .
$$

Therefore the classifying map $\varphi$ is the rational map given by the full linear system of quadrics contained in the ideal of $C \subset \mathbb{P}_{\omega}^{4}$. In fact the locus of non semistable extensions is exactly represented by $C$, as the next lemma shows.

Lemma 5.1.1 [Ber92] Let $(e)$ be an extension class in $\mathbb{P}_{\omega}^{4}$ and $\operatorname{Sec}(C)$ the secant variety of $C \subset \mathbb{P}_{\omega}^{4}$, then the vector bundle $E_{e}$ is not semistable if and only if $e \in C$ and it is not stable if and only if $e \in \operatorname{Sec}(C)$.

Remark 5.1.2 One can say even more. In fact, given $x, y \in C$ the secant line $\overline{x y}$ is the fiber of $\varphi$ over the S-equivalence class of $\omega(-x-y) \oplus \omega^{-1}(x+y)$.

This implies directly the following Corollary.

Corollary 5.1.3 The image of the secant variety $\operatorname{Sec}(C)$ by the classifying map $\varphi$ is the Kummer surface $K^{0} \subset|2 \Theta|$.

The hyperelliptic involution $\lambda$ acts on the canonical line bundle over $C$ and on its spaces of sections. A straightforward Riemann-Roch computation shows that $h^{0}\left(C, \omega^{3}\right)^{*}=5$. Then let $\pi: C \rightarrow \mathbb{P}^{1}$ be the hyperelliptic map. Then there is a canonical linearization for the action of $\lambda$ on $\omega$ that comes from the fact that $\omega=\pi^{*} \mathcal{O}_{\mathbb{P}^{1}}(1)$. In fact, by Kempf's Theorem ([DN89], Théorème 2.3), a line bundle on $C$ descends to $\mathbb{P}^{1}$ if and only if the involution acts trivially on the fibers over Weierstrass points. Thus we choose the linearization $\delta: \lambda^{*} \omega \stackrel{\sim}{\rightarrow} \omega$ that induces the identity on the fibers over Weierstrass points. This means that 


$$
\operatorname{Tr}\left(\lambda: L_{w_{i}} \rightarrow L_{w_{i}}\right)=1
$$

for every Weierstrass point $w_{i}$. Moreover we have that $d \lambda_{w_{i}}=-1$, which implies, via the Atiyah-Bott-Lefschetz fixed point formula ([GH78], p.421), that

$$
h^{0}\left(C, \omega^{3}\right)_{+}-h^{0}\left(C, \omega^{3}\right)_{-}=3 .
$$

Since $h^{0}\left(C, \omega^{3}\right)_{+}+h^{0}\left(C, \omega^{3}\right)_{-}=5$, this means that $h^{0}\left(C, \omega^{3}\right)_{+}=4$ and $h^{0}\left(C, \omega^{3}\right)_{-}=1$ and we can see that

$$
H^{0}\left(C, \omega^{3}\right)_{-}=\sum_{i=1}^{6} w_{i}
$$

Furthermore, we have

$$
E_{\lambda(e)}=\lambda^{*} E_{e}
$$

thus the points of $\mathbb{P}_{\omega+}^{3}:=\mathbb{P} H^{0}\left(C, \omega^{3}\right)_{+}^{*}$ will represent involution invariant extension classes. We will be particularly interested in the closed subset of $\mathbb{P}_{\omega+}^{3}$ parametrizing non-stable bundles, that is the variety $\operatorname{Sec}(C) \cap \mathbb{P}_{\omega+}^{3}$.

Lemma 5.1.4 The degree of $\operatorname{Sec}(C) \cap \mathbb{P}_{\omega+}^{3} \subset \mathbb{P}_{\omega+}^{3}$ equals 4. The hyperplane $\mathbb{P}_{\omega+}^{3}$ is everywhere tangent to $\operatorname{Sec}(C)$.

Proof: We recall that $C$ is embedded in $\mathbb{P}_{\omega}^{4}$ as a sextic curve. We also recall that that $\operatorname{dim}(\operatorname{Sec}(C))=3$ thus it is contained in $\mathbb{P}_{\omega}^{4}$ as a hypersurface. We project away from a general line in $\mathbb{P}_{\omega}^{4}$ onto a $\mathbb{P}^{2}$. Let

$$
\lambda: \mathbb{P}_{\omega}^{4}-\rightarrow \mathbb{P}^{2}
$$

be this projection, then the degree $\operatorname{deg}(\operatorname{Sec}(C))$ is given by the number of nodes of $\lambda(C) \subset \mathbb{P}^{2}$. Since $C$ doesn't intersect the general line in $\mathbb{P}_{\omega}^{4}$, the arithmetic genus of $\lambda(C)$ is 10 . This implies that $\lambda(C)$ has 8 nodes, so $\operatorname{deg}(\operatorname{Sec}(C))=8$. Now we want to compute the number of intersections of a general $\mathbb{P}^{1}$ contained in $\mathbb{P}_{\omega+}^{3}$ with $\operatorname{Sec}(C) \cap \mathbb{P}_{\omega+}^{3} \subset \mathbb{P}_{\omega+}^{3}$. Suppose that the line cuts in a point $z$ the secant $\overline{p q}$, with $p, q \in C$. A Riemann-Roch computation gives that $h^{0}\left(\omega^{3}(-p-q-\lambda(p)-\lambda(q))=2\right.$ so $p, q, \lambda(p)$ and $\lambda(q)$ are coplanar. This implies that the two secants $\overline{p q}$ and $\overline{\lambda(p) \lambda(q)}$ intersect in the point $z$, i.e. $\operatorname{deg}\left(\operatorname{Sec}(X) \cap \mathbb{P}_{\omega+}^{3}\right) \leq \frac{8}{2}=4$. Furthermore $p, q, \lambda(p), \lambda(q)$ are the only points of $C$ such that $h^{0}\left(\omega^{3}(-p-q-\lambda(p)-\lambda(q))=2\right.$. This means that there can't be another secant $\overline{h k}$, different from $\overline{p q}$ and $\overline{\lambda(p) \lambda(q)}$, passing through $z$. Hence $\operatorname{deg}\left(\operatorname{Sec}(C) \cap \mathbb{P}_{\omega+}^{3}\right)=4$ and $\mathbb{P}_{\omega+}^{3}$ is everywhere tangent to $\operatorname{Sec}(C)$. $\square$

In the following, we will denote

$$
W^{\prime}:=\operatorname{Sec}(X) \cap \mathbb{P}_{\omega+}^{3}
$$


and $\mathcal{W}:=\left\{w_{1}, \ldots, w_{6}\right\}$ will denote the set of the six Weierstrass points. Considering the fact that $C \cap \mathbb{P}_{\omega+}^{3}=\mathcal{W}$ and that $C \subset \operatorname{Sing}(\operatorname{Sec}(C))$ we can deduce that $W^{\prime}$ is a quartic surface in $\mathbb{P}_{\omega+}^{3}$ singular at the six points and containing the $\left(\begin{array}{l}6 \\ 2\end{array}\right)=15$ lines joining pairs of points of $\mathcal{W}$. Moreover, a partition of $\mathcal{W}$ in two subsets of cardinality 3 defines a pair of different $\mathbb{P}^{2} \subset \mathbb{P}_{\omega+}^{3}$, each one containing three of the six Weierstrass points. There are $\frac{1}{2}\left(\begin{array}{l}6 \\ 3\end{array}\right)=10$ such partitions and to each such partition one can associate the $\mathbb{P}^{1}$ obtained as intersection of the two $\mathbb{P}^{2}$ s. We will denote by $\mathbb{P}_{123}^{2}$ the $\mathbb{P}^{2}$ containing $w_{1}, w_{2}$ and $w_{3}$ and $\mathbb{P}_{456}^{2}$ the $\mathbb{P}^{2}$ containing $w_{4}, w_{5}$ and $w_{6}$. Furthermore we will denote by $\mathbb{P}_{123}^{1}=\mathbb{P}_{456}^{1}$ the line obtained as intersection of $\mathbb{P}_{123}^{2} \cap \mathbb{P}_{456}^{2}$.

Proposition 5.1.5 The surface $W^{\prime}$ contains the 10 lines $\mathbb{P}_{i j k}^{1}$, for any subset

$$
\{i, j, k\} \subset \mathcal{W}
$$

of cardinality 3.

Proof: We will prove the Proposition for $\mathbb{P}_{123}^{1}$, as for the other lines the proof is the same. By duality a $\mathbb{P}^{2} \subset \mathbb{P}_{\omega+}^{3}$ can be seen as a divisor in $\left|\omega^{3}\right|_{+}$. Notably $\mathbb{P}_{123}^{2}$ is associated to the divisor $D_{123}:=2 w_{1}+2 w_{2}+2 w_{3}$ and $\mathbb{P}_{456}^{2}$ to $D_{456}:=2 w_{4}+2 w_{5}+2 w_{6}$. Let

$$
\rho: \mathbb{P}_{\omega}^{4} \rightarrow \mathbb{P}^{2}
$$

be the projection away from $\mathbb{P}_{123}^{1}$. If the restriction of $\rho$ to $C$ gives a map of degree bigger than 1 , then $\mathbb{P}_{123}^{1} \subset W^{\prime}$. Moreover we denote by $\kappa$ the theta characteristic $\omega^{-1}\left(+w_{1}+w_{2}+w_{3}\right)$. The annihilator of the line $\mathbb{P}_{123}^{1}$ in $\left|\omega^{3}\right|$ is the linear subsystem

$$
\operatorname{Sym}^{2}\left(H^{0}(C, \omega \kappa)\right)=\left\langle D_{123}, D_{456}, \sum_{i=1}^{6} w_{i}\right\rangle .
$$

Furthermore we have

$$
C \cap \mathbb{P}_{\omega+}^{3}=\mathcal{W}
$$

and so $\mathbb{P}_{123}^{1} \cap C=\emptyset$. This implies that, once restricted to $C, \rho$ is a morphism. Let $Y \subset \mathbb{P} \operatorname{Sym}^{2}\left(H^{0}(C, \omega \kappa)\right)^{*}$ be the image of $C$. Then the following diagram commutes

$$
\begin{gathered}
C \\
\alpha \downarrow \searrow \rho \\
\mathbb{P}^{1}=|\omega \kappa| \hookrightarrow Y \subset \mathbb{P} \operatorname{Sym}^{2}\left(H^{0}(C, \omega \kappa)\right)^{*}
\end{gathered}
$$

where the vertical arrow $\alpha$ is the 3:1 map given by the linear system $|\omega \kappa|$. This means that the morphism $\rho$ is of degree 3 and $Y$ is a plane conic. It remains to prove that all secants of $C$ do not meet in one point of $\mathbb{P}_{123}^{1}$. Let us suppose that such a point $x \in \mathbb{P}_{123}^{1}$ exists and let us project $C$ from $x$. Let $\pi_{x}$ be the projection and $Z$ the image of $C$. Then $Z$ is a 
non degenerate curve in $\mathbb{P}^{3}$ and $\operatorname{deg}(Z) \cdot \operatorname{deg}\left(\pi_{x}\right)=6$; moreover, since we suppose all secants pass through $x, \operatorname{deg}\left(\pi_{x}\right) \geq 2$. As $Z$ is non degenerate, the only case we have to check is $\operatorname{deg}(Z)=3$ and $\operatorname{deg}\left(\pi_{x}\right)=2$, but by Castelnuovo's Lemma then $Z$ is the twisted cubic. Then the projection $\pi_{x}$ is the composition

$$
C \stackrel{2: 1}{\longrightarrow} Z \hookrightarrow \mathbb{P} S y m^{3} H^{0}(C, \omega)^{*}
$$

of the canonical map with the $3^{\text {rd }}$ Veronese. This implies that our $\mathbb{P}^{3}$ is isomorphic to $\mathbb{P}$ Sym $^{3} H^{0}(C, \omega)^{*} \cong \mathbb{P}_{\omega+}^{3}$, but this is absurd, as $x \in \mathbb{P}_{\omega+}^{3}$. This means at least one secant to $C$ intersects $\mathbb{P}_{123}^{1}$ in each point, that implies that $\mathbb{P}_{123}^{1} \subset W^{\prime}$.

Let us consider the Picard surface $\operatorname{Pic}^{1}(C)$ endowed with the Riemann theta divisor $\Theta$. It is well known that the Abel-Jacobi map

$$
\begin{aligned}
A J: C & \longrightarrow \operatorname{Pic}^{1}(C), \\
p & \mapsto \mathcal{O}_{C}(p),
\end{aligned}
$$

induces an isomorphism $C \cong \Theta \subset P i c^{1}(C)$. We also have the following exact sequence

$$
0 \longrightarrow \mathcal{O}_{P_{i c^{1}}(C)}(2 \Theta) \longrightarrow \mathcal{O}_{P_{i c^{1}}(C)}(3 \Theta) \longrightarrow \mathcal{O}_{\Theta}(3 \Theta) \longrightarrow 0 .
$$

Then the adjunction formula gives $\mathcal{O}(\Theta)_{\mid C \cong \Theta}=\omega_{C}$. Since $h^{1}\left(\operatorname{Pic}^{1}(C), 2 \Theta\right)=0$, taking global sections we have the following exact sequence

$$
0 \longrightarrow H^{0}\left(\operatorname{Pic}^{1}(C), 2 \Theta\right) \longrightarrow H^{0}\left(\operatorname{Pic}^{1}(C), 3 \Theta\right) \stackrel{r e s_{\mid \Theta}}{\longrightarrow} H^{0}\left(C, \omega^{3}\right) \longrightarrow 0 .
$$

This means that we have a surjective restriction map

$$
\operatorname{res}_{3 \Theta}: H^{0}\left(\operatorname{Pic}^{1}(C), 3 \Theta\right) \longrightarrow H^{0}\left(C, \omega^{3}\right) .
$$

Now the Abel-Jacobi map 32 embeds $C$ in $\operatorname{Pic}^{1}(C)$ as the theta divisor and the images of the Weierstrass points are the 6 odd theta characteristics. Furthermore we remark that

$$
\tau_{\mid \Theta \cong C}=\lambda: C \longrightarrow C
$$

Moreover we have chosen linearizations on $C$ and $\operatorname{Pic}^{1}(C)$ that are compatible, in the sense that the following diagram commutes.

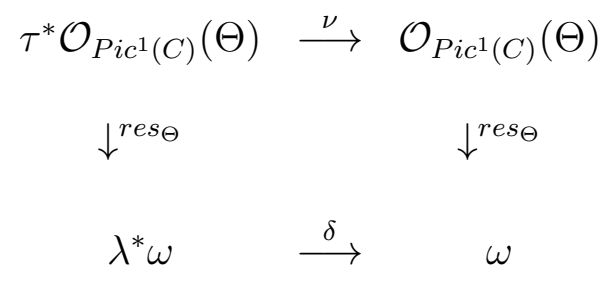


This means that the restriction morphisms respect the decomposition into eigenspaces of $H^{0}\left(\operatorname{Pic}^{1}(C), 3 \Theta\right)$ and $H^{0}\left(C, \omega^{3}\right)$. Moreover, since all sections of $\mathcal{O}_{P i c^{1}(C)}(2 \Theta)$ are invariant and the only section of $\mathcal{O}_{P i c^{1}(C)}(\Theta)$ is anti-invariant, the image of $H^{0}\left(\operatorname{Pic}^{1}(C), 2 \Theta\right)$ in $H^{0}\left(P i c^{1}, 3 \Theta\right)$ is contained in the anti-invariant subspace. This gives the following exact sequence

$$
0 \longrightarrow H^{0}\left(\operatorname{Pic}^{1}(C), 2 \Theta\right) \longrightarrow H^{0}\left(\operatorname{Pic}^{1}(C), 3 \Theta\right)_{-} \longrightarrow H^{0}\left(C, \omega^{3}\right)_{-} \longrightarrow 0 .
$$

This means also that there is an isomorphism

$$
M: H^{0}\left(P i c^{1}(C), 3 \Theta\right)_{+}^{*} \stackrel{\sim}{\longrightarrow} H^{0}\left(C, \omega^{3}\right)_{+}^{*} .
$$

Remark 5.1.6 As a birational model of $K^{1}$, the surface $W$ contains an interesting set of rational curves. It has in fact six double points at the image of the odd theta characteristics and the image of the theta divisor $\Theta \subset K^{1}$ is the only twisted cubic passing through these six nodes. The other 15 divisors of $K^{1}$ obtained as $t_{a}^{*} \Theta$, for $a \in J a c(C)[2]$ (see section 1 for the definition of $t_{a}$ ) are sent to the fifteen lines that pass through pairs of nodes. The ten even theta characteristics are blown up and the exceptional divisors are the ten lines obtained by intersecting two 2-planes in $|3 \Theta|_{+}^{*}$ each containing three nodes.

Moreover we have the following lemma.

Lemma 5.1.7 Let $F$ and $F^{\prime}$ be two quartic surfaces. If $F$ and $F^{\prime}$ contain 25 distinct lines, then $F \cong F^{\prime}$.

Proof: Two quartic surfaces in $\mathbb{P}^{3}$ coincide or intersect in a curve of degree 16 , but such a curve can't contain all the 25 lines the two surfaces share, thus they coincide.

Now, the identification

$$
\mathbb{P}(M):|3 \Theta|_{+}^{*} \longrightarrow \mathbb{P}_{\omega+}^{3}
$$

sends the images of the odd theta characteristics of $\operatorname{Pic}^{1}(C)$ to the images of the Weierstrass points of $C$. This means that, by Lemma 5.1.7, under the identification $\mathbb{P}(M)$ we have $W \cong W^{\prime}$. This in turn implies our second main result, i.e. Theorem 0.0.2.

Remark 5.1.8 The six double points of $W$ correspond to non semistable extension classes,

\subsection{A commutative diagram}

In the last section of this paper we want to show that not only the two surfaces $W^{\prime}$ and $W$ coincide but they are part of a larger commutative diagram which involves the duality map of the Kummer surface. First of all we will examine the following rational map

$$
\begin{aligned}
S: S y m^{2} C & -\rightarrow W^{\prime} \\
x+y & \mapsto \overline{x y} \cap \mathbb{P}_{\omega+}^{3} .
\end{aligned}
$$


Lemma 5.2.1 The rational map $S$ factorizes through the quotient $S y m^{2} C / \lambda$ and the induced rational map is finite of degree 1.

Proof: Recall from the proof of Lemma 5.1.4 that two secants $\overline{x y}$ and $\overline{p q}$ intersect $\mathbb{P}_{\omega+}^{3}$ in the same point if and only if $x=\lambda(p)$ and $y=\lambda(q)$. This directly implies the assertion and the fact that the induced rational map is of degree 1 .

Remark 5.2.2 Note that the exceptional locus of the map is given by the symmetric products of Weierstrass points.

We will call $S_{\lambda}: S y m^{2} C / \lambda \rightarrow W^{\prime}$ the induced map. We also have a morphism from $S y m^{2} C$ to $K^{0}$, defined in the following way

$$
\begin{aligned}
\varepsilon: \operatorname{Sym}^{2} C & \longrightarrow K^{0}, \\
x+y & \mapsto \omega(-x-y) .
\end{aligned}
$$

Note that also this map factorizes through the quotient $S y m^{2} C / \lambda$ since $(\omega(-x-y))^{-1} \equiv$ $\omega(-\lambda(x)-\lambda(y))$. Let us denote by

$$
\varepsilon_{\lambda}: \operatorname{Sym}^{2} C / \lambda \rightarrow K^{0}
$$

the induced morphism and by $\varepsilon_{\lambda}^{-1}: K^{0} \rightarrow S y m^{2} C / \lambda$ its birational inverse. This allows us to state the following Proposition.

Proposition 5.2.3 The composed map

$$
N: K^{0} \stackrel{\varepsilon_{\lambda}}{\rightarrow} S y m^{2} C / \lambda \stackrel{S_{\lambda}}{\rightarrow} W^{\prime} \stackrel{\varphi}{\rightarrow} \rightarrow K^{0}
$$

is the identity on a Zariski open set.

Proof: Let $U$ be the Zariski open set of $K^{0}$ complementary to the 16 symmetric theta divisors. We show that $M_{\mid U}=I d_{\mid U}$. Let $x, y \in C$ such that $\omega(-x-y) \sim \omega(-\lambda(x)-\lambda(y))$ is contained in $U$. By looking at Remark 5.1.2 one sees that $N(\omega(-x-y))$ is the S-equivalence class of $\omega(-x-y) \oplus \omega(-\lambda(x)-\lambda(y))$

We give now an analogue of Proposition 4.2.2 for $\operatorname{Pic}^{1}(C)$ and the line bundle $\mathcal{O}_{P i c^{1}(C)}(\Theta)$.

Proposition 5.2.4 Let $\Theta$ be the Riemann theta divisor on Pic ${ }^{1}(C)$. There is a canonical injection

$$
Q_{\Theta}: H^{0}\left(\operatorname{Pic}^{1}(C), 2 \Theta\right)^{*} \hookrightarrow \operatorname{Sym}^{2} H^{0}\left(\operatorname{Pic}^{1}(C), 3 \Theta\right)_{+}
$$

whose image is the space of quadrics in $|3 \Theta|_{+}^{*}$ passing through the six odd theta characteristics. 
Remark 5.2.5 The proof of Proposition 5.2.4 is analogue to that of Proposition 4.2.2. Furthermore, once we evaluate $Q_{\Theta}\left(H^{0}\left(\operatorname{Pic}^{1}(C), 2 \Theta\right)^{*}\right) \subset \operatorname{Sym}^{2} H^{0}\left(\operatorname{Pic}^{1}(C), 3 \Theta\right)_{+}$in $H^{0}\left(\operatorname{Pic}^{1}(C), 6 \Theta\right)_{+} \cong$ Sym $^{3} H^{0}\left(P_{i c}^{1}(C), 2 \Theta\right)$ one obtains the 4-dimensional subspace of polar cubics of $K^{1}$.

Moreover we have the following Lemma.

Lemma 5.2.6 [Kum00] The linear restriction map

$$
\text { res }: H^{0}\left(\mathbb{P}_{\omega}^{4}, \mathcal{I}_{C} \otimes \mathcal{O}(2)\right) \rightarrow H^{0}\left(\mathbb{P}_{\omega+}^{3}, \mathcal{O}(2)\right)
$$

is injective and its image is the space of quadrics on $\mathbb{P}_{\omega+}^{3}$ contained in the ideal of the 0 dimensional scheme $\mathcal{W}$.

We are now ready to state the main result of this section

Theorem 5.2.7 Let

$$
\mathcal{D}: K^{1} \rightarrow K^{0}
$$

be the duality birational map given by polar cubics and

$$
\chi: K^{1} \rightarrow \mathbb{P}_{\omega+}^{3}
$$

the rational map given by the linear system $|3 \Theta|_{+}$and the identification $\mathbb{P}(M)$, then $\chi=$ $S \circ \varepsilon_{\lambda}^{-1} \circ \mathcal{D}$ as rational maps.

Proof:We recall from Theorem 0.0.2 that $\chi$ and $S \circ \varepsilon_{\lambda}^{-1} \circ \mathcal{D}$ have the same image in $\mathbb{P}_{\omega+}^{3}=$ $|3 \Theta|_{+}$, that is the Weddle surface $W$. Then we remark that by Lemma 5.2.6 and Remark 5.2.5 the composition of $\chi$ with the restriction of $\varphi$ to $\mathbb{P}_{\omega+}^{3}$ gives the duality map on $K^{1}$. We obtain the same rational map (at least on an open subset) by composing $\mathcal{D}$ and $N$; since all the maps we are considering are generically one to one this implies the assertion.

Theorem 5.2.7 makes then the following diagram commute.

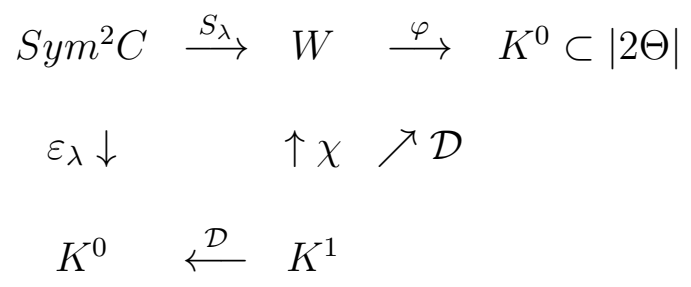

The classifying map $\varphi$ also defines a conic bundle over $\mathbb{P}^{3} \cong|2 \Theta|$. In fact for a general point $p \in \mathbb{P}^{3}$ the pre-image $\varphi^{-1}(p)$ consists of the intersection of three quadrics, that means $C$ plus a conic. Morover, let $S \subset \mathbb{P}_{\omega}^{4}$ be the cone over the twisted cubic $X \subset \mathbb{P}_{\omega+}^{3}$, in [Bol06] we have proven the following theorem. 
Theorem 5.2.8 Let $B l_{S} \mathbb{P}_{\omega}^{4}$ be the blow-up of $\mathbb{P}_{\omega}^{4}$ along the cone $S$ and $\mathbb{P}_{\mathcal{O}}^{3}$ the blow-up of $\mathbb{P}^{3} \cong|2 \Theta|$ in the point of $K^{0}$ corresponding to the origin. Let moreover $B l_{\mathcal{O}} K^{0}$ be the Blow-up of the Kummer surface $K^{0}$ in the origin. Then $\varphi: \mathbb{P}_{\omega}^{4--\rightarrow} \mathbb{P}^{3}$ resolves to a morphism

$$
\tilde{\varphi}: B l_{S} \mathbb{P}_{\omega}^{4} \longrightarrow \mathbb{P}_{\mathcal{O}}^{3}
$$

Furthermore the morphism $\tilde{\varphi}$ is a conic bundle whose degeneration locus is the surface $B l_{\mathcal{O}} K^{0} \subset \mathbb{P}_{\mathcal{O}}^{3}$.

\section{References}

[AR96] Allan Adler and S. Ramanan, Moduli of abelian varieties, Lecture Notes in Mathematics, vol. 1644, Springer-Verlag, Berlin, 1996.

[BB66] W. L. Baily, Jr. and A. Borel, Compactification of arithmetic quotients of bounded symmetric domains, Ann. of Math. (2) 84 (1966), 442-528.

[Bea88] Arnaud Beauville, Fibrés de rang 2 sur une courbe, fibré déterminant et fonctions thêta, Bull. Soc. Math. France 116 (1988), no. 4, 431-448.

[Bea91] _ Fibrés de rang deux sur une courbe, fibré déterminant et fonctions thêta. II, Bull. Soc. Math. France 119 (1991), no. 3, 259-291.

[Ber92] A. Bertram, Moduli of rank-2 vector bundles, theta divisors, and the geometry of curves in projective space, J. Differential Geom. 35 (1992), no. 2, 429-469.

[Bol06] Michele Bolognesi, Surfaces de weddle et leurs espaces de modules, Ph.D. thesis, Université de Montpellier II, 2006.

[Bur92] H. Burkhardt, Untersuchungen aus dem Gebiete der hyperelliptischen Modulfunktionen. I, Math. Ann. 41 (1892), no. 3, 313-343.

[Cob17] A. B. Coble, Point sets and allied Cremona groups i, ii, iii, Trans.AMS 18 (1917), $331-372$.

[DN89] J.-M. Drezet and M. S. Narasimhan, Groupe de Picard des variétés de modules de fibrés semi-stables sur les courbes algébriques, Invent. Math. 97 (1989), no. 1, 53-94.

[FSM04] Eberhard Freitag and Riccardo Salvati Manni, The Burkhardt group and modular forms. II, Transform. Groups 9 (2004), no. 3, 237-256.

[GH78] P. Griffiths and J. Harris, Principles of algebraic geometry, Wiley-Interscience [John Wiley \& Sons], New York, 1978, Pure and Applied Mathematics. 
[GH04] B. H. Gross and J. Harris, On some geometric constructions related to theta characteristics, Contributions to automorphic forms, geometry, and number theory, Johns Hopkins Univ. Press, Baltimore, MD, 2004, pp. 279-311.

[Har95] J. Harris, Algebraic geometry, Graduate Texts in Mathematics, vol. 133, SpringerVerlag, New York, 1995, A first course.

[HT84] J. Harris and L. W. Tu, On symmetric and skew-symmetric determinantal varieties, Topology 23 (1984), no. 1, 71-84.

[Hud90] R. W. H. T. Hudson, Kummer's quartic surface, Cambridge Mathematical Library, Cambridge University Press, Cambridge, 1990, With a foreword by W. Barth, Revised reprint of the 1905 original.

[Hun96] B. Hunt, The geometry of some special arithmetic quotients, Lecture Notes in Mathematics, vol. 1637, Springer, Berlin, 1996.

[Igu64] J.-I. Igusa, On the graded ring of theta-constants. I, Amer. J. Math. 86 (1964), 219-246.

[Igu72] — Theta functions, Springer-Verlag, New York, 1972, Die Grundlehren der mathematischen Wissenschaften, Band 194.

[Kum00] C. Kumar, Invariant vector bundles of rank 2 on hyperelliptic curves, Michigan Math. J. 47 (2000), no. 3, 575-584.

[Mum66] D. Mumford, On the equations defining abelian varieties. I, Invent. Math. 1 (1966), $287-354$.

[Mum74] _ Prym varieties. I, Contributions to analysis (a collection of papers dedicated to Lipman Bers), Academic Press, New York, 1974, pp. 325-350.

[NR69] M. S. Narasimhan and S. Ramanan, Vector bundles on curves, Algebraic Geometry (Internat. Colloq., Tata Inst. Fund. Res., Bombay, 1968), Oxford Univ. Press, London, 1969, pp. 335-346.

[SD74] B. Saint-Donat, Projective models of $K-3$ surfaces, Amer. J. Math. 96 (1974), 602-639.

[vdG87] G. van der Geer, Note on abelian schemes of level three, Math. Ann. 278 (1987), no. $1-4,401-408$.

Michele Bolognesi

Institut de Mathématiques et de Modélisation de Montpellier

Université Montpellier II

Case Courrier 051 
Place Eugène Bataillon

34095 Montpellier Cedex 5

E-mail: bolo@math.univ-montp2.fr 